\title{
Cytoskeleton-Associated Risk Modifiers Involved in Early and Rapid Progression of Sporadic Creutzfeldt-Jakob Disease
}

\author{
Saima Zafar ${ }^{1}$ - Neelam Younas ${ }^{1} \cdot$ Nadeem Sheikh $^{2} \cdot$ Waqas Tahir $^{1} \cdot$ Mohsin Shafiq $^{1}$. \\ Matthias Schmitz $^{1} \cdot$ Isidre Ferrer $^{3,4}$. Olivier Andréoletti ${ }^{5} \cdot$ Inga Zerr $^{1}$
}

Received: 19 January 2017 / Accepted: 28 April 2017

(C) Springer Science+Business Media New York 2017

\begin{abstract}
A high priority in the prion field is to identify pre-symptomatic events and associated profile of molecular changes. In this study, we demonstrate the pre-symptomatic dysregulation of cytoskeleton assembly and its associated cofilin-1 pathway in strain and brain region-specific manners in MM1 and VV2 subtype-specific Creutzfeldt-Jakob disease at clinical and pre-clinical stage. At physiological level, $\mathrm{PrP}^{\mathrm{C}}$ interaction with cofilin-1 and phosphorylated form of cofilin ( $\mathrm{p}$-cofilin(Ser3)) was investigated in primary cultures of mouse cortex neurons (PCNs) of $\operatorname{PrP}^{\mathrm{C}}$ wildtype and knockout mice $\left(\mathrm{PrP}^{-/-}\right)$. Short-interfering RNA downregulation of active form of cofilin-1 resulted in the redistribution/downregulation of $\operatorname{PrP}^{\mathrm{C}}$, increase of activated form of microglia, accumulation of dense form of $\mathrm{F}$ actin, and upregulation of p-cofilin(Ser3). This upregulated
\end{abstract}

Saima Zafar and Neelam Younas have equal contributions.

Significance Statement In prion field, a high demand is arising to uncover pre-clinical events and the alteration of associated pathway as the clinical stage is much squatter than pre-clinical stage. For public health concern, early diagnosis is a big need as the disease can be transmitted by blood. So, if the blood donors had prion disease in a pre-clinical stage, and the prion disease was diagnosed at a later date, this could be a risk for public health and a concern to develop diagnostic tools by studying early pre-clinical molecular pathways. To address this need, targeting cofilin-1 activity in LIMK-APP-SSH1 signaling pathways might be a promising strategy to study the most likely disease progression and the internal homeostasis of misfolded proteins.

Saima Zafar

sz_awaan@yahoo.com; saima.zafar@med.uni-goettingen.de

1 Department of Neurology, Clinical Dementia Center, and DZNE, Georg-August University, University Medical Center Goettingen (UMG), Robert-Koch-Str. 40, 37075 Goettingen, Germany

2 Department of Zoology, University of the Punjab, Lahore, Pakistan
p-cofilin(Ser3) showed redistribution of expression predominantly in the activated form of microglia in PCNs. At pathological level, cofilin-1 expression was significantly altered in cortex and cerebellum in both humans and mice at pre-clinical stage and at early symptomatic clinical stage of the disease. Further, to better understand the possible mechanism of dysregulation of cofilin-1, we also demonstrated alterations in upstream regulators; LIM kinase isoform 1 (LIMK1), slingshot phosphatase isoform 1 (SSH1), RhoA-associated kinase (Rock2), and amyloid precursor protein (APP) in sporadic Creutzfeldt-Jakob disease MM1 mice and in human MM1 and VV2 frontal cortex and cerebellum samples. In conclusion, our findings demonstrated for the first time a key pre-clinical response of cofilin-1 and the associated pathway in prion disease.
3 Institute of Neuropathology, IDIBELL-University Hospital Bellvitge, University of Barcelona, Hospitalet de Llobregat, Spain

4 Network Center for Biomedical Research of Neurodegenerative Diseases (CIBERNED), Institute Carlos III, Ministry of Health, Madrid, Spain

5 Institut National de la Recherche Agronomique/Ecole Nationale Vétérinaire, Toulouse, France 
Keywords Cofilin · Actin · CJD · MM1 - VV2 · Prion protein $\cdot$ LIMK $\cdot$ Rock $\cdot$ APP $\cdot$ SSH1 $\cdot$ Microglia $\cdot$ Cerebellum $\cdot$ Cortex

\section{Introduction}

Sporadic Creutzfeldt-Jakob disease (sCJD) is the most common form of prion diseases in humans [1]. Based on pathological form of prion protein $\left(\mathrm{PrP}^{\mathrm{Sc}}\right)$ electrophoretic mobility and allelic variation at codon 129, sCJD has been divided into six subtypes [2-4] among which SCJD MM1 and SCJD VV2 are the two most commonly occurring subtypes [3]. Although clinico-pathological features of the molecular disease subtypes differ markedly by the affected brain area, severity, and morphological features; but spongiform degeneration or severe gliosis, neuronal loss, and dysfunction are critical and constant features of CJD [5]. Synaptic loss and degeneration of the axon terminal are early abnormalities in experimental scrapie $[6,7]$. Indeed, accumulation of pathological form $\left(\mathrm{PrP}^{\mathrm{Sc}}\right)$ of prion triggers a pathological mechanism leading to synaptic abnormalities [5]. Recently, a role of $\operatorname{PrP}^{\mathrm{C}}$ in control of neurite sprouting and dendritic spine formation has been described through the modulation of actin dynamics $[8,9]$.

Actin cytoskeleton is crucial for neurite growth cone, spine development, and formation of synapses in neurons [10-12]. Perfect balance in remodeling of actin is required for maturation and maintenance of the synapses by regulating spine morphology [13-17]. In mammals, the actin-depolymerizing factor (ADF)/cofilin family of proteins, consisting of cofilin-1 (a nonmuscle type), cofilin-2 (a muscle-type), and ADF (also known as destrin), is the major and critical regulator of actin dynamics and reorganization by depolymerization and severing of actin filaments [18-20]. A growing body of evidence provides support to a link between neurite transportation abnormalities and synaptic plasticity impairment observed in neurodegenerative diseases [21], as well as alterations in the organization and dynamics of the actin cytoskeleton initiated by cofilin-1 [19, 22-25]. The regulatory response of cofilin- 1 activity is quite complex and is maintained by a cohort of kinases and phosphatases [26]. In particular, cofilin-1 is inactivated by LIM kinase isoform 1 (LIMK1)-mediated phosphorylation at serine 3 (Ser3) that halts its actin binding activity, and dephosphorylation by slingshot phosphatase isoform 1 (SSH1), reactivates cofilin-1 [19, 25, 27-29]. Although other kinases and phosphatases can also affect cofilin-1 activity, LIMK1 and SSH1 show the highest substrate specificity [26]. Remarkably, phosphorylation of LIMK1 at threonine 505 and 508 activates it, and phosphorylation at serine 937 and 978 inactivates SSH1 [30]. Further, not only cofilin-1 but also LIMK1 is dephosphorylated by SSH1 activity. So, SSH1 can control cofilin-1 activity either directly or indirectly through an upstream regulation of LIMK1 [30]. Moreover, SSH1 is also a potential interactor of
14-3-3 protein [31], and 14-3-3 is known to be markedly elevated in the CSF of CJD patients [32-34].

In this study, our main objective was to uncover molecular changes in cofilin-LIMK pathway, a major regulator of actin dynamics, in two most frequent subtypes of SCJD (MM1 and VV2) and in a mouse model at pre-symptomatic and symptomatic stages of the disease. Interaction between $\operatorname{PrP}^{\mathrm{C}}$ and cofilin-1, as well as its phosphorylated form, was investigated in cellular model. This study demonstrated that regulation of cofilin-LIMK pathway is altered in SCJD in a brain regionand subtype-specific manner. Remarkably, dysregulation of cofilin-LIMK pathway at pre-symptomatic stage of the disease signifies events initiated early in the pathological cascade. Meanwhile, translation study in primary cultures of mouse cortex neurons (PCNs) demonstrated the functional aspect of cofilin-1 depletion on actin dynamics, microglial activation, and upregulation/redistribution of phosphorylated form of cofilin at clinical stage of the disease in humans. Further differential manifestation/regulation of cofilin-LIMK pathway in both subtypes of sCJD provides potential new insights into the molecular mechanisms involved in pathology and heterogeneity of prion diseases.

\section{Materials and Methods}

\section{Ethics Statement}

Human samples from the Institute of Neuropathology Brain Bank (HUB-ICO-IDIBELL Biobank) and Biobank of Hospital Clinic-IDIBAPS were obtained following the Spanish legislation (Ley de la Investigación Biomédica 2013 and Real DecretoBiobancos 2014) and the approval of the local ethics committees.

All animal experiments were performed in accordance with the ethical standard set by Regierungspräsidium Tübingen (Regional Council) Experimental No. FLI 231/07 file reference number 35/9185.81-2. All animal experiments have been performed in compliance with the institutional and French national guidelines, in accordance with the European Community Council Directive 86/609/EEC. The experimental protocol was approved by the INRA Toulouse/ENVT ethics committee.

\section{CJD Patients, Samples Processing, and Subtype Characterization}

In this study, 30 pathologically confirmed SCJD patient's brain tissue samples, i.e., frontal cortex and cerebellum regions (15 of each MM1 and VV2 subtypes) and 15 age-matched control cases $(\mathrm{CON})$ were analyzed. All 45 samples were obtained from the Institute of Neuropathology Brain Bank (HUBICO-IDIBELL Biobank) and Biobank of Hospital Clinic- 

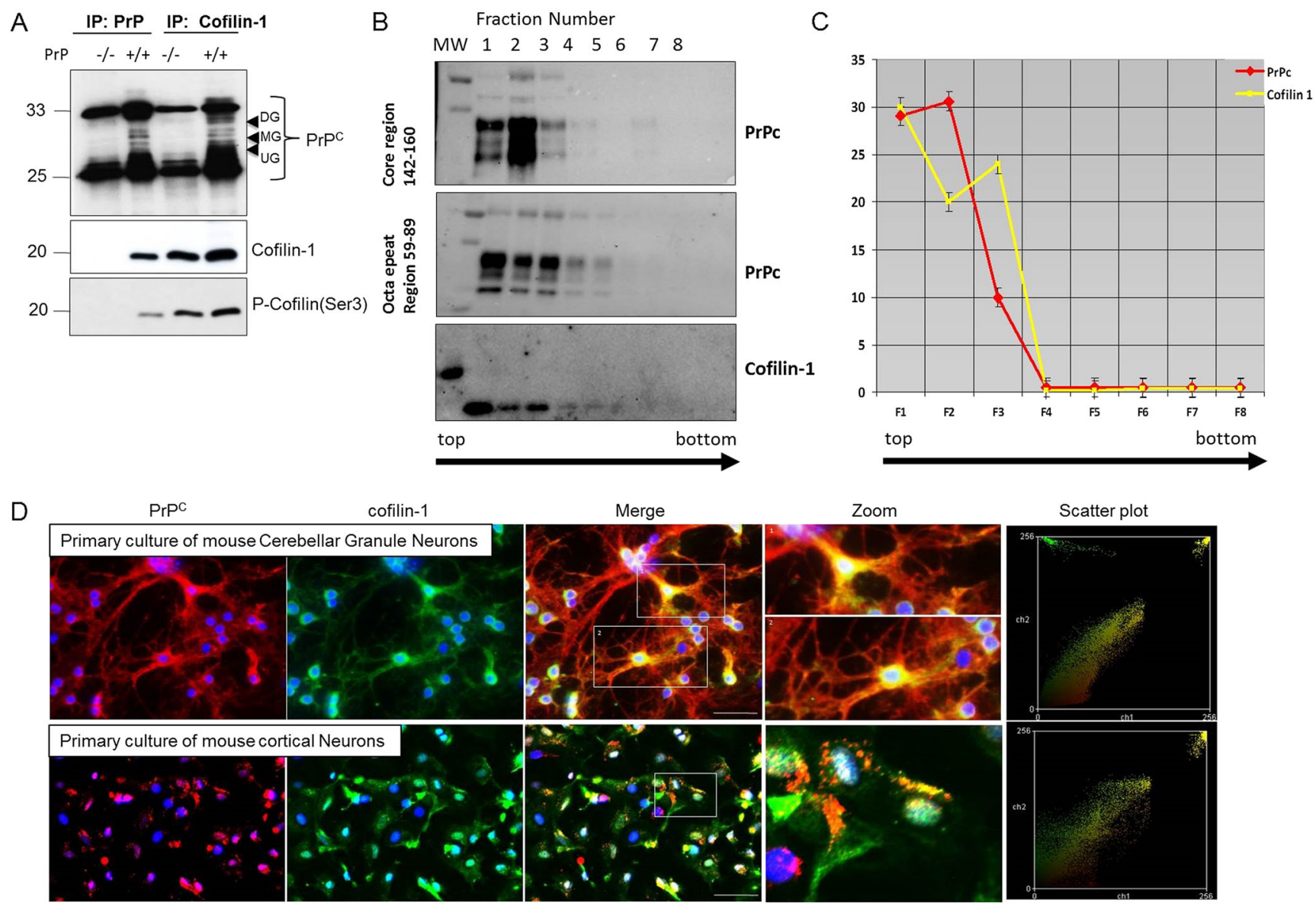

$\mathrm{F}$

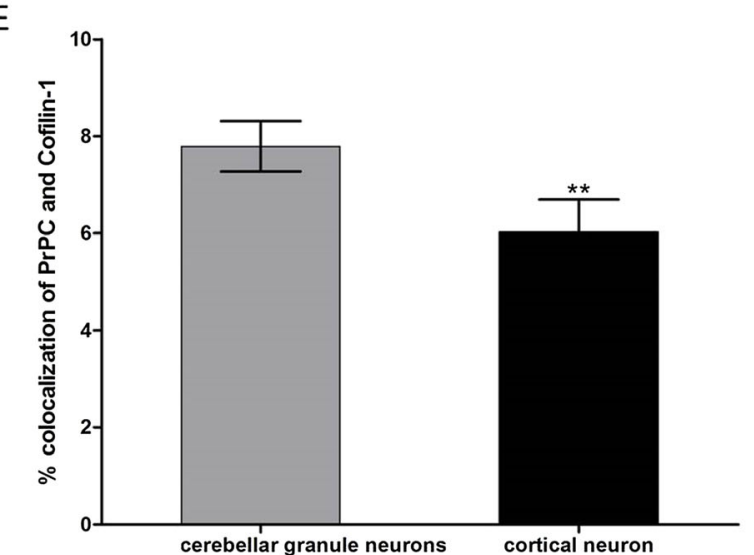

Fig. 1 Interactive association between $\operatorname{PrP}^{\mathrm{C}}$ and cofilin-1. Primary cultures of mouse cortex neurons (PCNs) encoding PrP gene and knockout $\left(\mathrm{PrP}^{--}\right)$were prepared from pregnant mice at embryonic day 14. a Total cellular lysate co-immunoprecipitated (IP) with $\mathrm{PrP}^{\mathrm{C}}$ antibody (lane $1 \mathrm{PrP}^{-1-} \mathrm{PCNs}$ and lane $2 \mathrm{PrP}^{\mathrm{C}}$-expressing $\mathrm{PCNs}$ ); cofilin-1 antibody (lane $3 \mathrm{PrP}^{-/}$PCNs and lane $4 \mathrm{PrP}^{\mathrm{C}}$-expressing $\mathrm{PCNs}$ ); and then immunoblotted with SAF70 $\operatorname{PrP}^{\mathrm{C}}$, cofilin-1, and p-cofilin(Ser3) antibodies. b, c Western blot with $\mathrm{PrP}^{\mathrm{C}}$ and cofilin-1 from fractions isolated after ultracentrifugation using sucrose step gradient. Densitometry analysis from four independent $( \pm \mathrm{SD})$ sedimentation ultracentrifugation fractionation experiments. $\mathbf{d} \operatorname{PrP}^{\mathrm{C}}$ and cofilin-1

IDIBAPS. The mean age and gender of study cases were as described previously $[35,36]$. In brief, for sCJD frontal cortex,

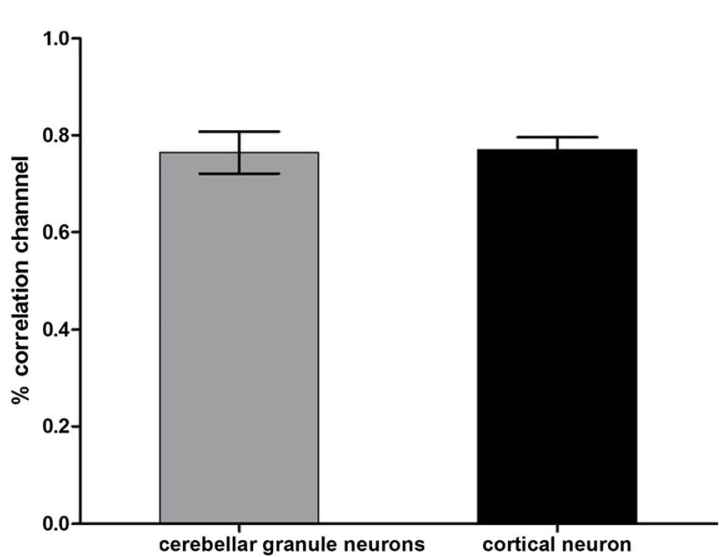

distribution was analyzed in primary cultures of mouse cerebellar granule neurons (PCGNs) and primary mouse corticle neurons (PCNs) using 3F4 anti-PrP ${ }^{\mathrm{C}}$ (red), anti-cofilin-1 (green) antibodies, and DAPI (blue-nuclear staining). At least 35 cells were observed per condition per experiment for an equal exposure time (Scale bar $10 \mu \mathrm{m})$. The co-localization scatter frequency plots of the individual pixels from paired images and co-localization frequency graphs were generated by ImageJ (WCIF plugin) software. e, f Pearson's colocalization correlation coefficient rp $(-1 \leq \mathrm{rp} \leq 1)$ showed \% co-localization and \% correlation btween $\operatorname{PrP}^{\mathrm{C}}$ and cofilin-1 in cerebellar granule neuron and in cortical neurons. Graph was generated by ImageJ (WCIF plugin) software

60 years of mean age in control $(10 \mathrm{M} / 5 \mathrm{~F}), 68$ years of mean age in SCJD MM1 $(10 \mathrm{M} / 5 \mathrm{~F})$, and 63 years of mean age in 

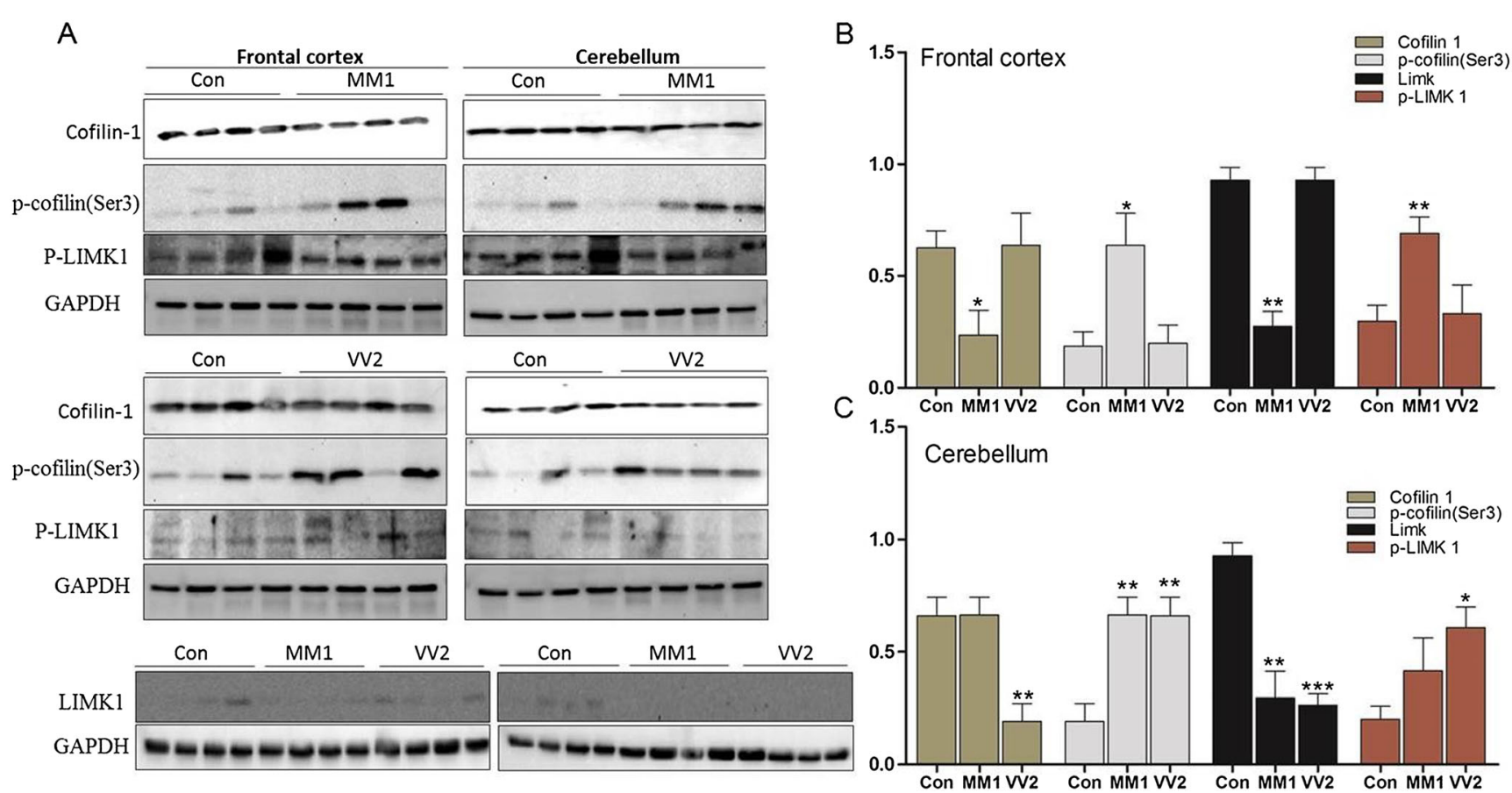

Fig. 2 Cofilin-1, p-cofilin(Ser3), LIMK1, and P-LIMK1 expressions in sCJD MM1 and VV2 subtypes. Western blotting analysis: a cofilin-1, pcofilin(Ser3), LIMK1, and P-LIMK1 in four representative cases each for control, sCJD MM1, and sCJD VV2 cases; GAPDH immunostaining was used to normalize total protein loading. b, c Densitometry analysis

from four independent $( \pm \mathrm{SD})$ immunoblotting experiments of 15 control (Con), $15 \mathrm{MM} 1$, and $15 \mathrm{VV} 2$ cases. The significance was calculated with one-way ANOVA Friedman test $(* P<0.05$, $* * P<0.01$, *** $P<0.001)$. $A U$ arbitrary units

sCJD VV2 (5 M/10F). In cerebellum, the mean age was 62 years in control $(11 \mathrm{M} / 4 \mathrm{~F}), 66$ years of mean age in sCJD MM1 (10M/5F), and 63 years of mean age in SCJD VV2 (4M/ 10F). After post-mortem interval ranging $1 \mathrm{~h} 45 \mathrm{~min}$ to $24 \mathrm{~h}$ $30 \mathrm{~min}$, coronal sections $1 \mathrm{~cm}$ thick were cut from one of the hemispheres. Along with selectively dissected areas of encephalon, coronal sections were rapidly frozen on metal plates over dry ice, sorted in separate bags, labeled with waterresistant ink and stored at $-80{ }^{\circ} \mathrm{C}$ until further use for biochemical investigations. The other hemisphere was fixed in $4 \%$ buffered formalin for 3 weeks for morphological studies and neuropathological examination and characterization. The analysis of the codon 129 genotype of PrP gene (Met: M or Val: V) was performed after isolation of genomic DNA from blood samples according to standard methods. Western blot profile of $\mathrm{PrP}^{\mathrm{Sc}}$ was classified as type 1 or type 2 based on electrophoretic mobility after proteinase $\mathrm{K}(\mathrm{PK})$ digestion.

\section{sCJD MM1 and VV2 Transgenic Mice}

Double transgenic Tg340 mice overexpressing about 4-fold level of human $\operatorname{PrP}^{\mathrm{C}}$ with methionine at codon 129 (Met129 and Val129) on a murine PrP knockout background were used, as described previously $[9,36,37]$. Inocula were prepared from SCJD MM1 and VV2 brain tissues as 10\% (w/v) homogenates. Individually identified 6- to 10 -week-old mice were anesthetized and inoculated with $2 \mathrm{mg}$ of brain

homogenate in the right parietal lobe using a 25-gauge disposable hypodermic needle (six animals per group and time point). Mice were observed daily, and the neurological status was assessed weekly. When disease progression was evident, or at the end of lifespan, animals were euthanized, necropsy was performed, and the brain was removed. A part of the brain was fixed by immersion in $10 \%$ buffered formalin to quantify spongiform degeneration and perform immunohistological procedures. The other part was frozen at $-80{ }^{\circ} \mathrm{C}$ to extract protein. Survival time was calculated for each isolate and expressed as the mean of the survival day post-inoculation (dpi) of all mice scoring positive for $\operatorname{PrP}^{\mathrm{Sc}}$. Infection rate was determined as the proportion of mice scoring positive for $\mathrm{PrP}^{\mathrm{Sc}}$ from all inoculated mice.

\section{Primary Culture of Mouse Cortex, Cerebellum, Gene Manipulation, Transfection, and RNAi}

PCNs and cerebellum (PCE) were prepared as described previously $[36,38]$. In brief, embryos were removed from pregnant mice at embryonic day 14 under halothane (Sigma) anesthesia; the mice were prnp (the PrP-encoding gene) and knockout $\left(\mathrm{PrP}^{-/-}\right)$[39]. Animals were killed by cervical dislocation and the dissected embryonic cortex was mechanically dissociated and plated on polyethylenimine $(1 \mathrm{mg} / \mathrm{ml})$-coated glass coverslips in culture wells. 
After 3 days in vitro (DIV), the medium was replaced with N5 medium [40] with $180 \mathrm{mg} / \mathrm{L}$ glucose and supplemented with 5\% FBS and 1\% FCS. Three micromolars of cytosine arabinoside was added to prevent astrocyte proliferation (resulting in at least 97\% pure neuronal cultures) and $1 \mu \mathrm{M}$ MK-801 to prevent excitotoxicity [41]. The medium was changed daily. On DIV 5, FCS was removed and the FBS content was reduced to $1 \%$.

The cells were seeded in complete DMEM prior to transient transfection with plasmid and short-interfering RNA (siRNA) duplexes using Lipofectamine 2000 (Invitrogen, Carlsbad, CA, USA) according to the manufacturer's instructions. Cofilin-1 siRNA duplex had a sense strand 5'-GGAG GACCUGGUGUUCAUC-3' [42], and non-targeting siRNA duplex (control siRNA duplex negative control: Eurogentec) [9] was used as a negative control.

\section{Assessment of Neuronal/Cell Viability and Caspase-3 Activity Assay}

The viability of neurons in primary cortical cultures, with and without depletion of cofilin-1 and in the presence of $\operatorname{PrP}^{\mathrm{C}}$, was checked by counting number of healthy nuclei per unit area after Hoechst staining. The identification of individual neuron viability that was unequivocally healthy on the basis of its nuclear appearance using a combination of fluorescent illumination and phase contrast [43].

Cell viability assay was performed as described previously [44]. Briefly, the adherent cells were grown up to $60-70 \%$ confluency and then detached from flasks using $1 \times$ TrypsinEDTA. The cells were spun down at $4{ }^{\circ} \mathrm{C}$ for $5 \mathrm{~min}$ at $400 \times g$ and resuspended in culture media. Cells were then dispensed into 24-well plates (Nunc, Roskilde, Denmark) at a final concentration of $1 \times 10^{5}$ cells/well and incubated for $12 \mathrm{~h}$. The culture media were then removed and replaced prior to MTS [3-(4,5-dimethylthiazol-2-yl)-5-(3-carboxymethoxyphenyl)2-(4-sulfophenyl) $2 \mathrm{H}$-tetrazolium, inner salt] treatment. The effect of the presence of $\operatorname{PrP}^{\mathrm{C}}$ and the treatment of TNFalpha on cell viability was measured using the MTS cell proliferation assay, which measures the reduction of MTS tetrazolium salt to formazan in metabolically active cells [45]. The cells were then treated with 1:20 ratio of MTS reagent (Promega Co. Madison, WI, USA) $2 \mathrm{mg} / \mathrm{ml}$ with $0.5 \%$ glucose and PMS $0.92 \mathrm{mg} / \mathrm{ml}$ with $0.5 \%$ glucose. Cells were incubated for $1 \mathrm{~h}$ at $37{ }^{\circ} \mathrm{C}$ for color development, and the absorbance values were read at $490 \mathrm{~nm}$ using a Multiscan Plate Reader (Labsystems, VA, USA) and Accent software 2.6. Background absorbance from controls was incubated in media with the MTS reagent and was subtracted from sample wells after the final absorbance was obtained.

The caspase-3-activity assay allows quantitative measurement of caspase-3 (DEVDase) protease activity, which is an early regulatory event in the apoptotic cell death process. The assay was performed using caspase-3 activity assay kit according to the manufacturer's recommendations. Briefly, cofilin-1 depleted PCNs were lysed in the cell lysis buffer for $15 \mathrm{~min}$ at $4{ }^{\circ} \mathrm{C}$, and this was followed by centrifugation at $10,000 \times g$. Protein concentration was estimated from the supernatants, and the total cell lysate $(50 \mu \mathrm{g})$ was then incubated with $50 \mu \mathrm{M}$ caspase-caspase-3-specific substrate DEVD-pNA (Asp-Glu-Val-Asp p-nitroanilide) for $4-5 \mathrm{~h}$ at $37{ }^{\circ} \mathrm{C}$. The caspase-3 inhibitor Z-vad-FMK $(20 \mu \mathrm{M})$ was used as control. Caspase-3-mediated release of pNA was measured by absorbance at $405 \mathrm{~nm}$. Background absorbance from the control (untreated cells) was subtracted from the samples after the final absorbance was obtained.

\section{Co-sedimentation Assay}

Two hundred microliters of $10 \%$ brain homogenate in PBS containing 2\% w/v sarkosyl ( $\mathrm{pH} 7.4$ ) were clarified for $5 \mathrm{~min}$ at $500 \times \mathrm{g}$, and were carefully layered onto the top of $10-60 \%$ sucrose step gradient. Gradients were prepared in thin wall polyallomer tubes $(11 \times 34 \mathrm{~mm}, 2.2 \mathrm{ml}$, Beckman $)$, and sucrose serial dilutions $(10,12.5,15,17.5,20,22.5$, and $60 \%, w / v)$ were made in PBS containing $1 \%$ sarkosyl (pH 7.4). All the gradients in an experiment were prepared with a same batch of buffers. For avoiding mixing of sucrose serial dilutions while setting gradient in tubes, tubes (sucrose layers) were frozen for $10 \mathrm{~min}$ at $-80^{\circ} \mathrm{C}$ after adding each sucrose layer; and next layer was then added over previous frozen layer. Ultracentrifugation was performed at $55,000 \mathrm{rpm}$ for $5 \mathrm{~h}$ at $5{ }^{\circ} \mathrm{C}$ in Optima ${ }^{\mathrm{TM}} \mathrm{TL}$ Ultracentrifuge (Beckman Coulter) equipped with Beckman Coulter TLS-55 rotor. These conditions corresponded to $\mathrm{RCF}=259,000 \times g$, angular velocity $\omega=5759.59 \mathrm{rad} / \mathrm{s}$, and $k$ factor $=50$. After centrifugation, 20 fractions, $100 \mu \mathrm{l}$ each, were collected carefully from top to bottom in separate tubes and proceeded for immunoblotting.

\section{Co-immunoprecipitation}

Cell lysis was performed as described previously $[9,46]$, and the insoluble cell debris was removed by centrifugation at $543,000 \times g$ for $15 \mathrm{~min}$ at $4{ }^{\circ} \mathrm{C}$. Immunoprecipitation was performed using magnetic Dynabeads protein $\mathrm{G}$, according to the manufacturer's instructions. Total cytoplasmic cell extracts or immunoprecipitated proteins (corresponding to $2 \times 10^{6}$ cells/ lane) were subjected to $12.5 \%$ 1-DE SDS-PAGE and, after transferring to polyvinylidenedifluoride membranes (Millipore), immunoblotting was performed as described above.

\section{Co-immunofluorescence Confocal Microscopy}

PCNs were platted on chambered slides [Lab-Tek ${ }^{\mathrm{TM}}$ II; Thermo Fisher Scientific (Nunc GmbH \& Co. KG), Langenselbold 
A

dpi

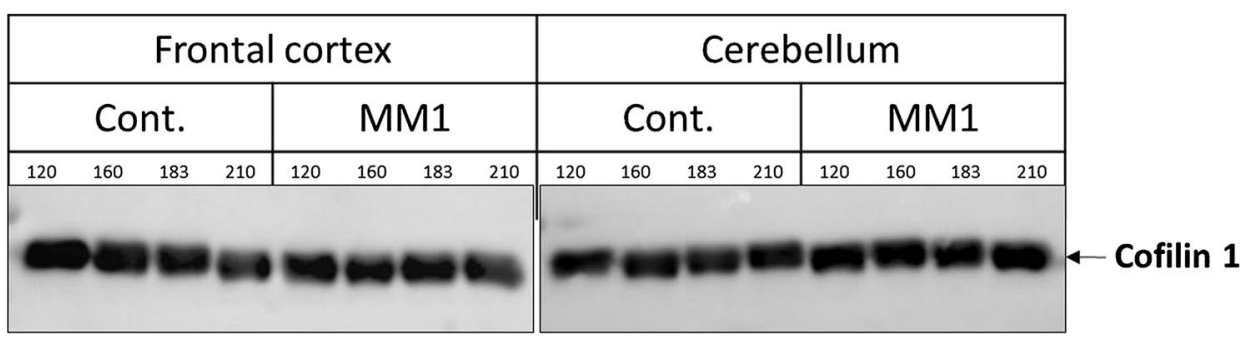

dpi

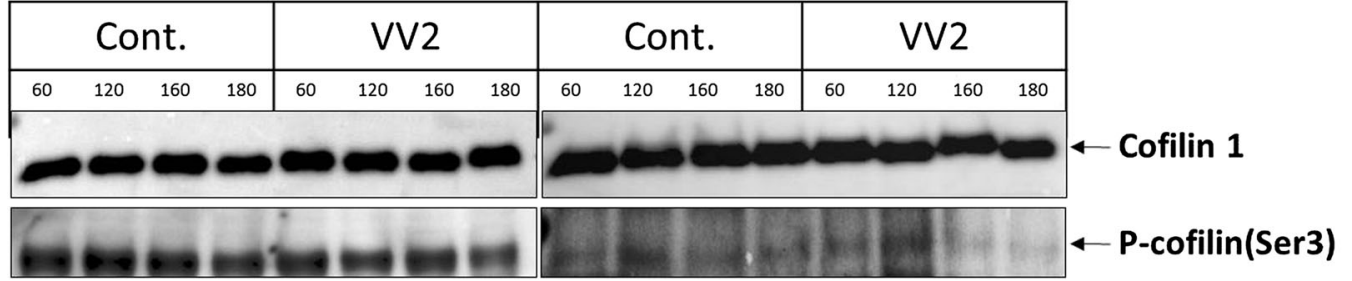

B

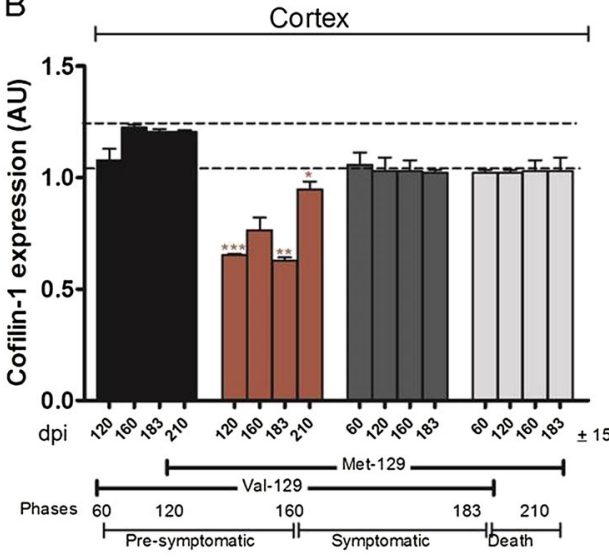

C

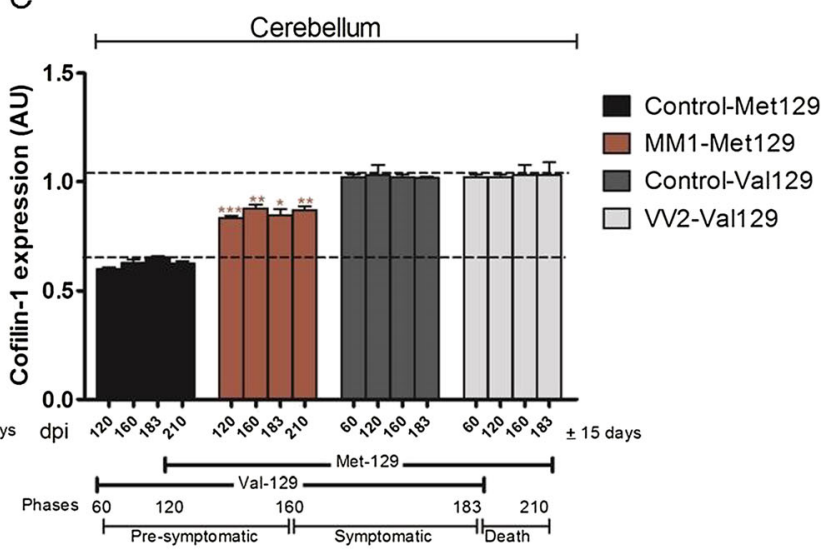

D

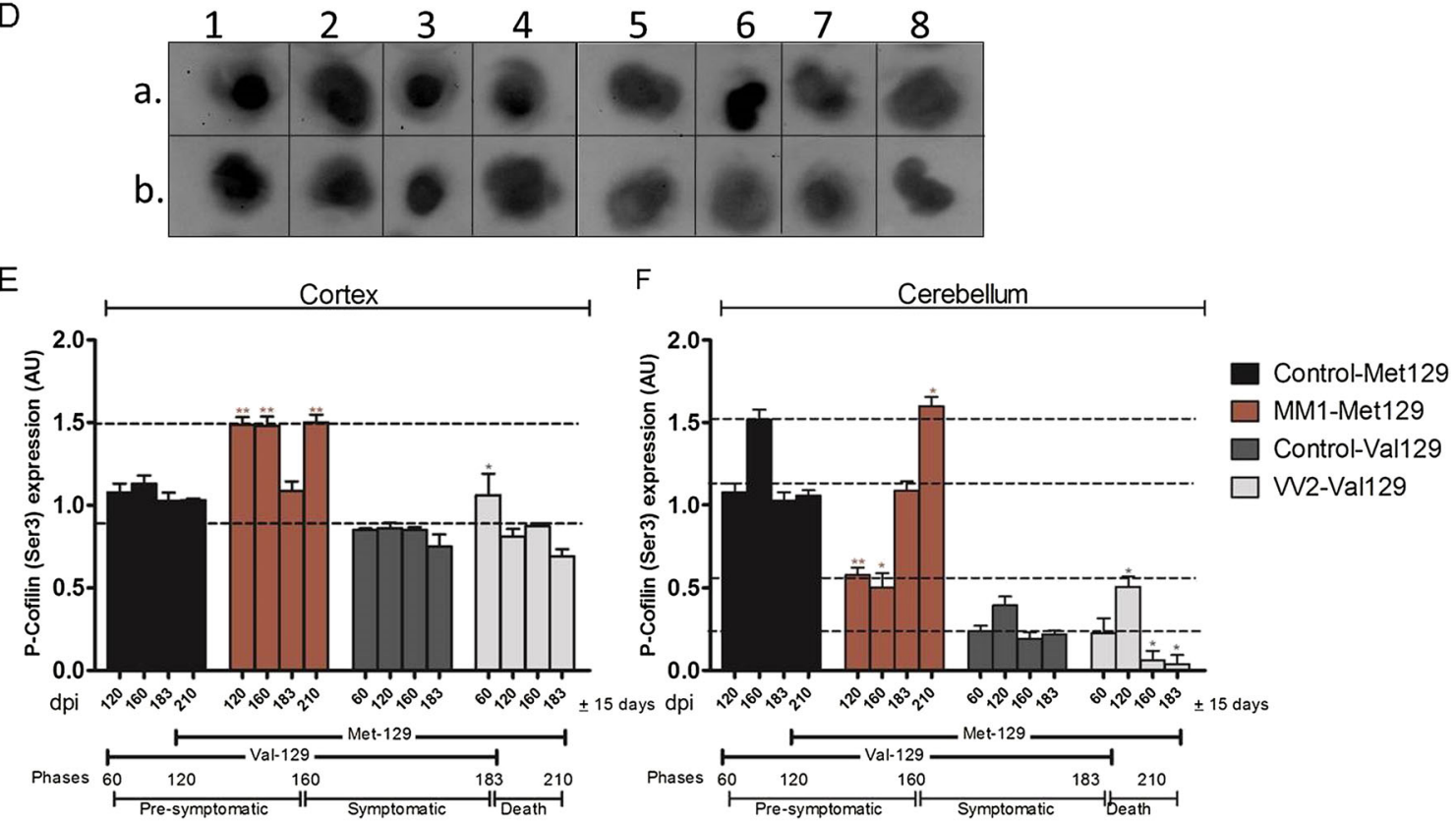

Site] and lipotransfected with cofilin-1 siRNA. Subsequently, they were washed in $1 \times$ PBS and then fixed for 15 min with $100 \%$ ethanol. After fixation, cells were permeabilized with
$0.2 \%$ Triton X-100 in $1 \times$ PBS, followed by a 20 -min blocking step using $0.2 \%$ casein-solution containing Tween 20 . Colocalization of F-actin with cofilin-1, $\operatorname{PrP}^{\mathrm{C}}$ with Iba1 and $\mathrm{p}$ - 
Fig. 3 Expression of cofilin-1 and p-cofilin(Ser3) at pre-symptomatic and symptomatic stage of sCJD MM1 and VV2 in mice. Tg340 mice expressing about 4-fold level of human PrPM129 were infected with sCJD MM1 and also VV2 10\% brain homogenates. Samples were collected at indicated days post-infection (dpi): pre-symptomatic stage (60 dpi in VV2, 120 dpi in MM1) and symptomatic stages (120183 dpi in VV2 and 160-210 dpi). a, d Cofilin and p-cofilin(Ser3) expression in the cortex and cerebellum were observed by Western blot analysis using cofilin and p-cofilin(Ser3) antibodies. b, c, e, f Densitometry analysis from four independent $( \pm \mathrm{SD})$ immunoblotting experiments of cofilin and p-cofilin(Ser3) from control and sCJD MM1- and VV2-infected PrPM129 mice in cortex and cerebellum. The significance was calculated with one-way ANOVA Friedman test $(* P<0.05)$

cofilin(Ser3), and cofilin-1 with p-cofilin(Ser3) was detected by exposure to the primary antibodies [anti-PrP $3 \mathrm{~F} 4$ $(1: 200)$, mouse anti-cofilin-1 (1:100), mouse anti-Factin (1:100), mouse anti- p-cofilin(Ser3) (1:100)] overnight at $4{ }^{\circ} \mathrm{C}$. The monoclonal antibodies were detected by incubating slides for $60 \mathrm{~min}$ with Alexa 488 conjugated anti-rabbit (1:200), Alexa 488 conjugated antimouse (1:200), or Cy3-labeled anti-mouse secondary antibody (1:200). Incubation with TO-PRO-3 iodide for $10 \mathrm{~min}$ was performed to visualize nuclei. Finally, coverslips were placed on the glass slides which were then mounted with Fluoromount (DAKO, Hamburg, Germany). All the steps were further carried out as described previously [9].

\section{Antibodies, Reagents, and Immunoblot Analysis}

Mouse anti-PrP mAb SAF32, mouse anti-PrP mAb SAF70 (1:1000; SpiBio, Paris, France), mouse anti-PrP 3F4 mAb (1:1000; Merck Millipore, Darmstadt, Germany), rabbit anticofilin mAb (1:1000; Sigma-Aldrich, Munich, Germany), rabbit anti-cofilin (phospho S3) mAb (1:500), mouse antiGAPDH mAb (1:1000), mouse anti-LIM kinase $1 \mathrm{mAb}$ (1:500), rabbit anti-LIM kinase 1 (phospho T508) pAb (1:500), rabbit anti-slingshot homolog $1 \mathrm{~L} \mathrm{pAb}(1: 1000)$, rabbit anti- RhoA-associated kinase (Rock2) pAb (1:500), rabbit anti-Iba1 mAb (1:1000), mouse anti-amyloid beta A4 protein $\mathrm{mAb}(1: 1000)$, and rabbit anti-calcineurin A mAb $(1 ; 1000)$ from Abcam, Cambridge, UK were used as primary antibodies. HRP-conjugated rabbit anti-mouse pAb (IBA, Goettingen, Germany), HRP-conjugated goat anti-rabbit pAb (Jackson ImmunoResearch Laboratories Europe, Ltd.), goat anti-mouse pAb cy3-conjugated (Dianova, Hamburg, Germany), anti-rabbit pAb (Santa Cruz Biotechnology, Santa Cruz, CA, USA), goat anti-rabbit (Alexa 488-conjugated), and anti-mouse (Alexa 488-conjugated) were used as secondary antibodies. Protease and phosphatase inhibitor cocktail (Roche, Mannheim, Germany), Hoechst 33342 (SigmaAldrich, Steinheim, Germany), halothane anesthesia, cytosine arabinoside (Sigma, St. Louis, MO, USA), MK-801
(Research Biochemicals International), penicillin, and streptomycin (Gibco) were also used.

Cell lysis and immunoblotting were performed as described previously $[36,46]$. Briefly, cells were lysed (50 mM Tris- $\mathrm{HCl}, \mathrm{pH} \mathrm{8,1 \%}$ Triton X-100, 0.5\% CHAPS, $1 \mathrm{mM}$ DTT), and lysates were cleared of cell debris ( $1 \mathrm{~min}$, $1000 \times g, 4^{\circ} \mathrm{C}$ ). Cell lysates were supplemented with protease and phosphatase inhibitors (Roche) and were separated on $12.5 \%$ 1-DE SDS-PAGE. Expression of proteins was analyzed by immunoblot using overnight exposure at $4{ }^{\circ} \mathrm{C}$ to anti-PrP SAF70 mAb (1:1000), anti-cofilin mAb (1:1000), anti-cofilin (Phospho S3) mAb (1:1000), anti-GAPDH mAb (1:1000), anti-LIM Kinase $1 \mathrm{mAb}$ (1:500), anti-LIM kinase 1 (Phospho T508) pAb (1:500), anti-slingshot homolog $1 \mathrm{~L} \mathrm{pAb}$ (1:1000), anti-Rock2 pAb (1:500), rabbit anti-Iba1 mAb (1:1000), anti-amyloid beta A4 protein $\mathrm{mAb}(1: 1000)$, and anti-calcineurin $\mathrm{mAb}(1 ; 1000)$. Membranes were then rinsed in $1 \times$ TBS-T and incubated with the corresponding horseradish peroxidase-conjugated secondary antibody (diluted 1:2000/1:5000) for $1 \mathrm{~h}$ at RT. Immunoreactivity was detected after immersion of the membranes into enhanced chemiluminescence (ECL) solution and exposure to ECL-Hyperfilm (Amersham Biosciences, Buckinghamshire, UK). Images were documented using the ScanMaker4 (Microtek, International), after correction for the background, and band intensities were determined by densitometry using Lab image (version 2.7.1, Kapelan GmbH, Germany) data analyzer software.

\section{Statistical Analysis}

All results in this study were obtained from at least four independent sets of experiments and were expressed as mean \pm S.D. using descriptive statistics. Densitometric analysis of 1-DE gels was performed using Labimage (version 2.7.1 Kapelan, Leipzig, Germany) software.

\section{Results}

\section{Selective Interactive Association Between $\operatorname{PrP}^{\mathrm{C}}$ and Cofilin-1}

Previous studies have shown an interaction between $\operatorname{PrP}^{\mathrm{C}} /$ $\operatorname{PrP}^{\mathrm{Sc}}$ and cofilin-1, but the role of cofilin-1 in the pathogenesis of Prion diseases is still not clear [46, 47]. So firstly, we were interested to explore interactive association between $\mathrm{PrP}^{\mathrm{C}}$ and cofilin-1. For this, we co-immunoprecipitated cofilin-1 from $\operatorname{PrP}^{\mathrm{C}}$ wild-type and $\operatorname{PrP}^{\mathrm{C}}$ knockout $\left(\operatorname{PrP}^{--}\right)$ primary cortical neurons ( $\mathrm{PCNs}$ ) using $\operatorname{PrP}^{\mathrm{C}}$ antibody by Dynabeads $\mathrm{G}$ protein-coupled magnetic beads, and purified eluates were co-immunoblotted with SAF $70 \operatorname{PrP}^{\mathrm{C}}$ antibody (Fig. 1a). No signal was detected using purified eluates from 
A

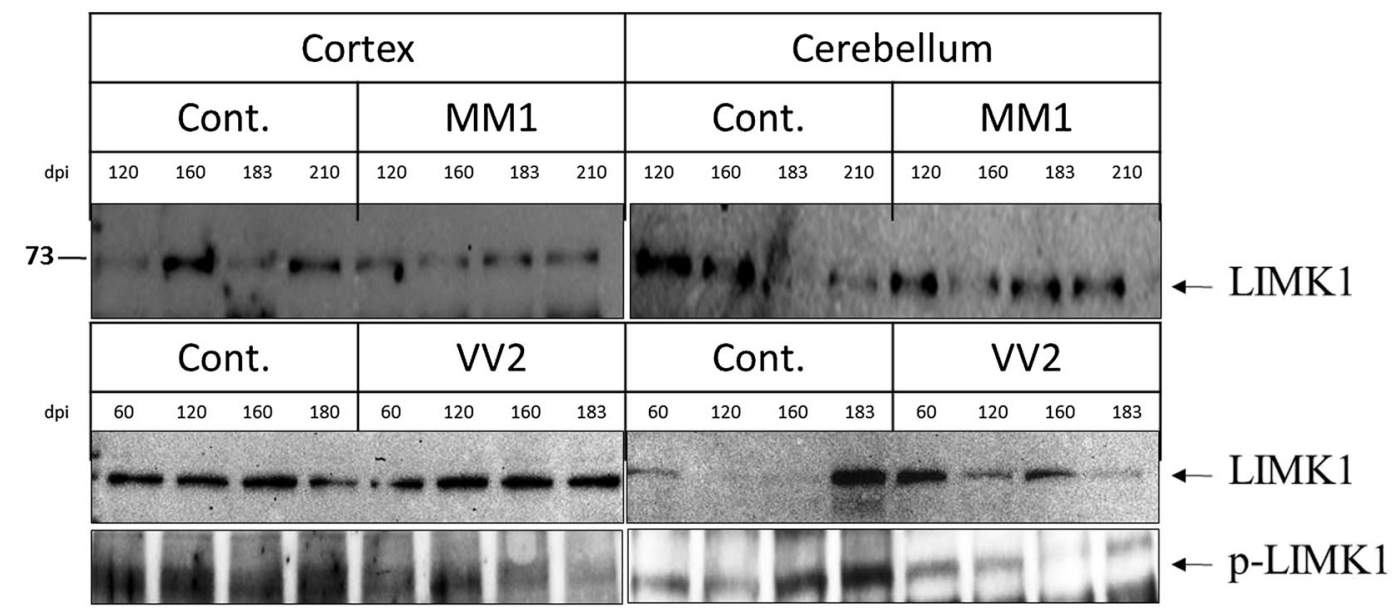

B

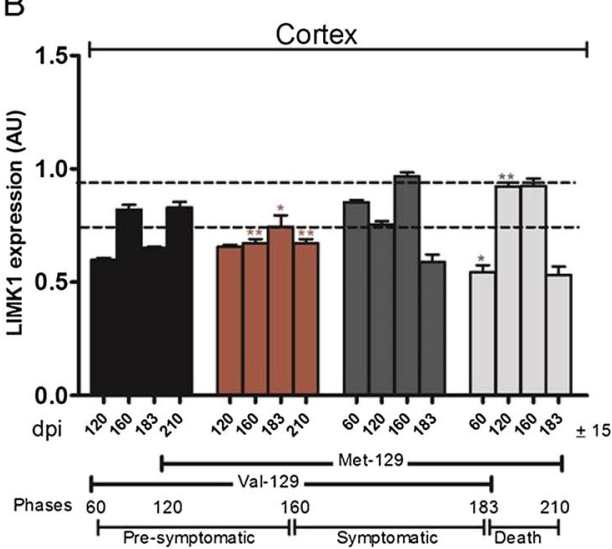

C

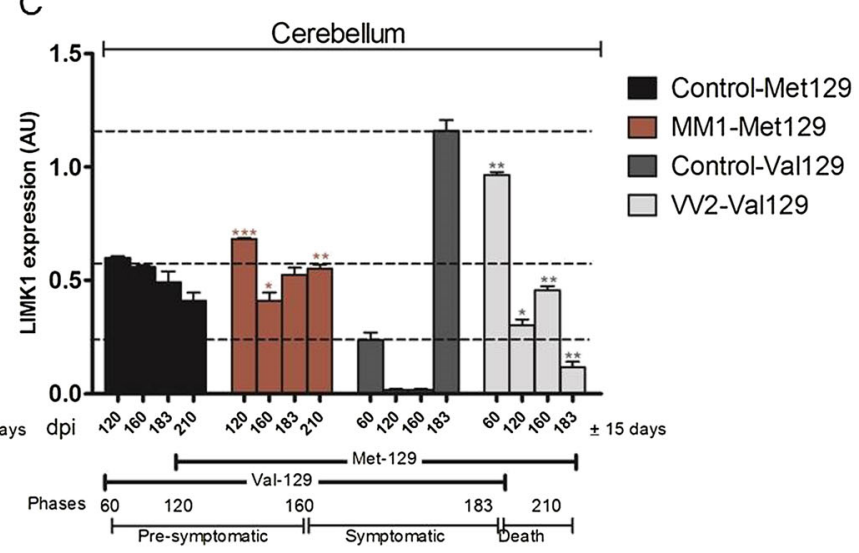

D

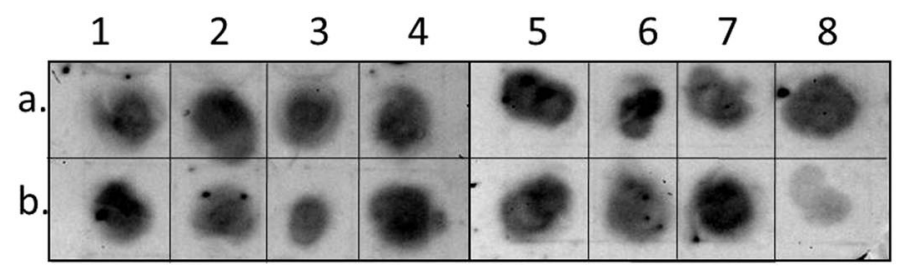

E

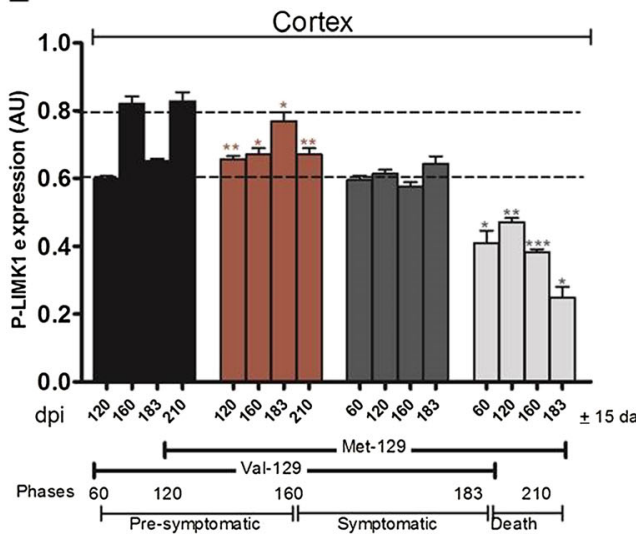

F

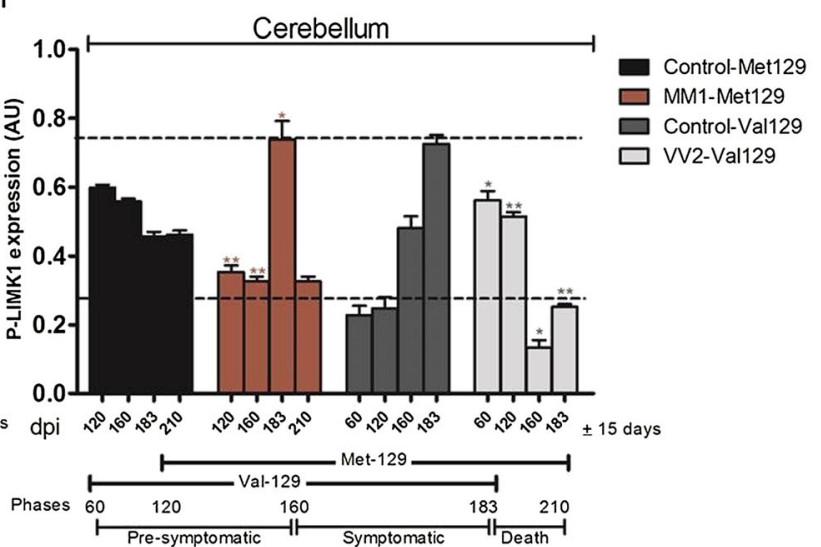

$\mathrm{PrP}^{-/-}$PCNs (Fig. 1a, b). In order to further confirm these observations, we co-immunoprecipitated $\mathrm{PrP}^{\mathrm{C}}$ using cofilin1 and p-cofilin antibodies (Fig. 1a). Eluates from this reverse co-immunoprecipitation were immunoblotted again with
SAF70 $\operatorname{PrP}^{\mathrm{C}}$ antibody to verify the $\operatorname{PrP}^{\mathrm{C}}$ interaction (Fig. 1a, b). Further, $\operatorname{PrP}^{\mathrm{C}}$-expressing primary cortical cultures were coimmunoprecipitated with $\operatorname{PrP}^{\mathrm{C}}$ and cofilin-1 antibodies followed by immunoblotting with anti-cofilin-1 antibody to 
Fig. 4 Expression of LIMK1 and P-LIMK1 at pre-symptomatic and symptomatic stage of sCJD MM1 and VV2 in mice. Tg340 mice expressing about 4-fold level of human PrPM129 were infected with sCJD MM1 and also VV2 10\% brain homogenates. Samples were collected at indicated days post-infection (dpi): pre-symptomatic stage (60 dpi in VV2, 120 dpi in MM1) and symptomatic stages (120183 dpi in VV2 and 160-210 dpi). a LIMK1 and P-LIMK1 expression in the cortex and cerebellum of SCJD MM1 and VV2 in mice were observed by Western blot analysis using LIMK1 and P-LIMK1 antibodies. $\mathbf{d}$ Dot blot analysis of P-LIMK1 in the cortex and cerebellum were observed by Western blot analysis using LIMK1 and P-LIMK1 antibodies. Frontal cortex (lane al = control 120 dpi, a2$=$ control $160 \mathrm{dpi}, a 3=$ control $183 \mathrm{dpi}, a 4=$ control $210 \mathrm{dpi}$, $b 1=$ sCJD MM1-infected $120 \mathrm{dpi}, b 2=$ sCJD MM1-infected $160 \mathrm{dpi}$, $b 3=$ sCJD MM1-infected $183 \mathrm{dpi}, b 4=$ sCJD MM1-infected $210 \mathrm{dpi}$ ). Cerebellum (lane $a 5=$ control $120 \mathrm{dpi}, a 6=$ control $160 \mathrm{dpi}, a 7=$ control $183 \mathrm{dpi}, a 8=$ control $210 \mathrm{dpi} ; b 5=$ sCJD MM1-infected $120 \mathrm{dpi}$, $b 6=$ sCJD MM1-infected $160 \mathrm{dpi}, b 7=$ sCJD MM1-infected $183 \mathrm{dpi}$, $b 8=$ sCJD MM1-infected 210 dpi). b, c, e, f Densitometry analysis from four independent $( \pm \mathrm{SD})$ immunoblotting experiments of LIMK1 and PLIMK1 from control and SCJD MM1- and VV2-infected PrPM129 mice in cortex and cerebellum. The significance was calculated with one-way ANOVA Friedman test $(* P<0.05)$

confirm the interaction (Fig. 1c). Likewise, confocal laser coimmunofluorescence scanning showed region-specific co-localization of $\operatorname{PrP}^{\mathrm{C}}$ and cofilin-1 in PCNs and in cerebellar neurons (PCB) (Fig. 1d-f). The Pearson's correlation coefficient data demonstrated significant less co-localization of $\operatorname{PrP}^{\mathrm{C}}$ with cofilin-1 in cortical neurons as compared to cerebellar granule neurons (Fig. 1e). However, correlation channel did not show significant altered response (Fig. 1f). The interactive association between $\operatorname{PrP}^{\mathrm{C}}$ and cofilin-1 was further verified with co-sedimentation assay at steady state and strikingly, $\operatorname{PrP}^{\mathrm{C}}$ and cofilin-1 shared identical fractions and demonstrated the selective functional association between cofilin-1 and $\operatorname{PrP}^{\mathrm{C}}$ (Fig. 1g, h).

\section{LIMK1 Pathway Mediated Alteration of Cofilin-1 Expression/Activity in CJD MM1 and VV2 Patients and in Pre-symptomatic and Symptomatic Stages of SCJD MM1 and VV2 Mice}

This interaction of cofilin-1 with $\operatorname{PrP}^{\mathrm{C}}$ suggests that there might be some functional correlation. So accordingly, we sought to explore molecular changes in cofilin-LIMK pathway in cerebellum and frontal cortex of two most frequent subtypes (MM1 and VV2) of SCJD, as well as age-matched controls (15 samples in each group). Total homogenates of the frontal cortex and cerebellum were analyzed using

A

\begin{tabular}{|c|c|c|c|c|c|c|}
\hline \multicolumn{3}{|c|}{ Frontal cortex } & \multicolumn{3}{|c|}{ Cerebellum } & \\
\hline Cont. & MM1 & VV2 & Cont. & MM1 & VV2 & \\
\hline-- & - & - - & & & $\rightarrow$ & ROCK2 \\
\hline $\mathrm{kes} \cdot \mathrm{me}=$ & $=$ & $\mathrm{e}$ & 14 & & $a=$ & APP \\
\hline
\end{tabular}

B

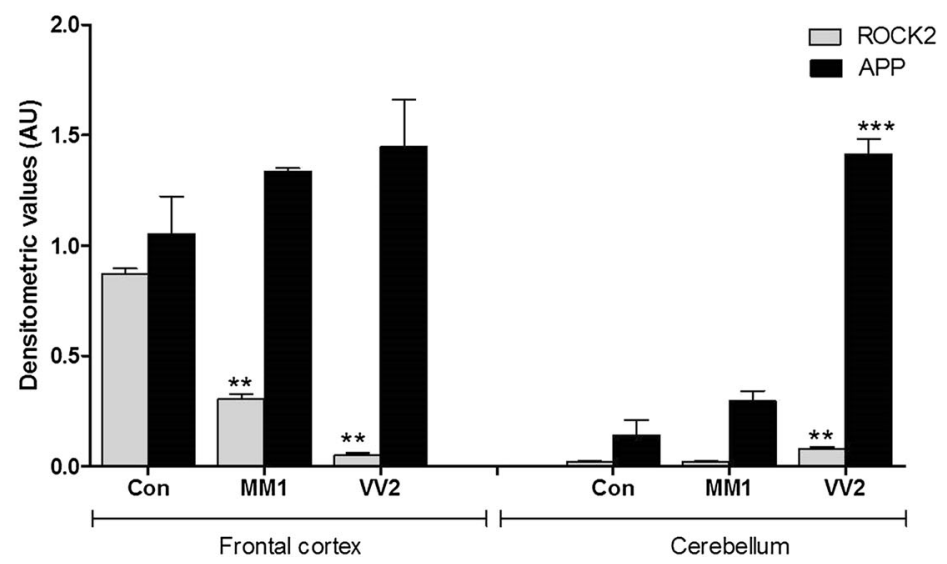

Fig. 5 Rock2 and APP in sCJD MM1 and VV2 subtypes. Western blotting analysis: a Rock2 and APP in four representative cases each for control, sCJD MM1, and sCJD VV2 cases; GAPDH immunostaining was used to normalize total protein loading. b
Densitometry analysis from four independent $( \pm \mathrm{SD})$ immunoblotting experiments of 15 control (Con), $15 \mathrm{MM} 1$, and $15 \mathrm{VV} 2$ cases. The significance was calculated with one-way ANOVA Friedman test $(* P<0.05, * * P<0.01, * * * P<0.001) . A U$ arbitrary units 


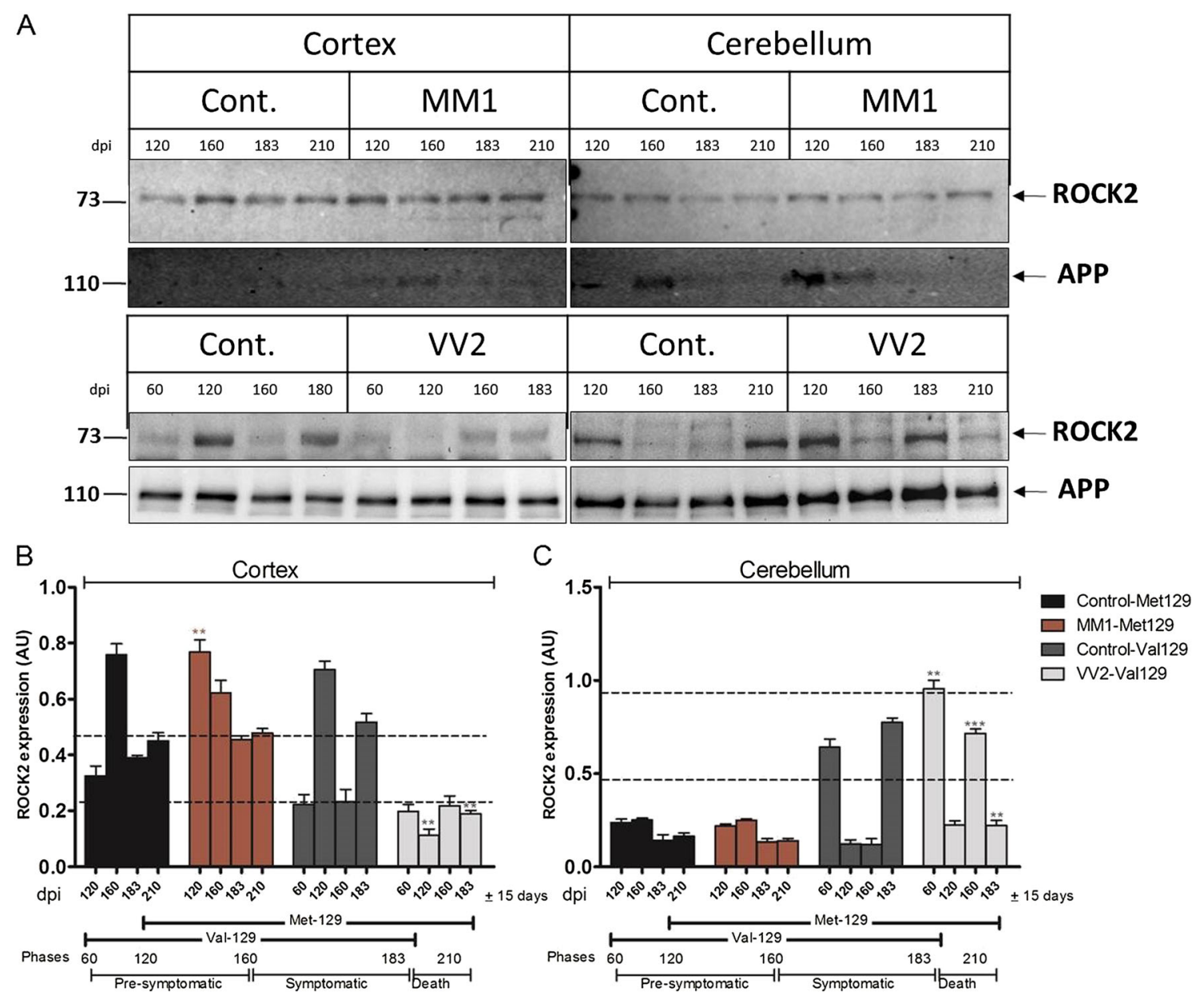

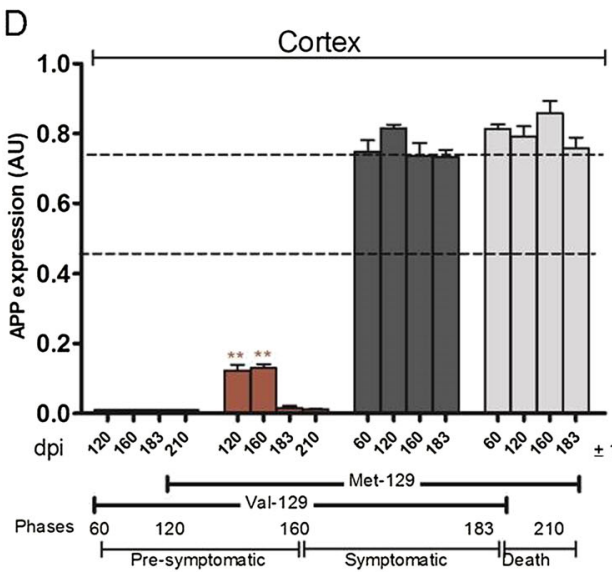

Fig. 6 Expression of Rock2 and APP at pre-symptomatic and symptomatic stage of sCJD MM1 and VV2 in mice. Tg340 mice expressing about 4-fold level of human PrPM129 were infected with sCJD MM1 and also VV2 10\% brain homogenates. Samples were collected at indicated days post-infection (dpi): pre-symptomatic stage (60 dpi in VV2, 120 dpi in MM1) and symptomatic stages (120183 dpi in VV2 and 160-210 dpi). a Rock2 and APP expressions in

cofilin-1 and p-cofilin(Ser3) monoclonal antibodies (Fig. 2). Densitometry analysis revealed significantly altered cofilin-1 expression at the protein level in the frontal

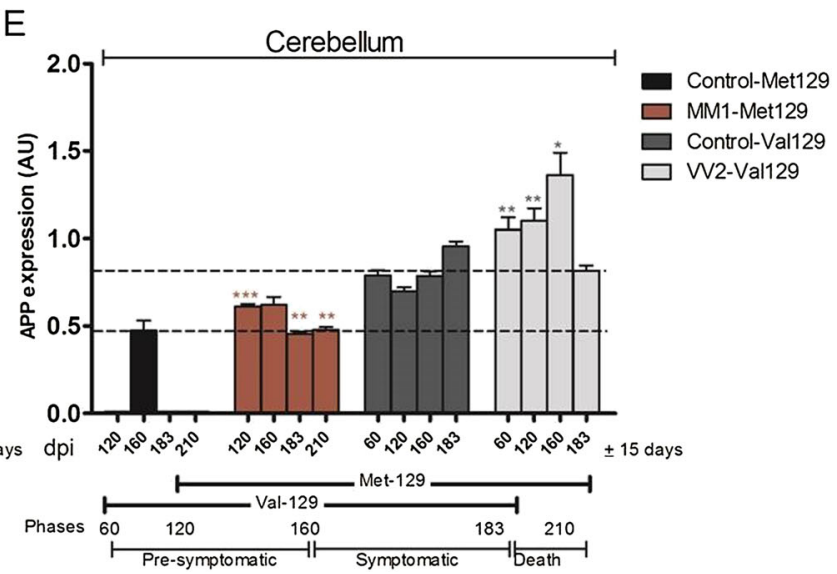

the cortex and cerebellum were observed by Western blot analysis using Rock2 and APP antibodies. b-e Densitometry analysis from four independent $( \pm \mathrm{SD})$ immunoblotting experiments of Rock2 and APP from control and SCJD MM1- and VV2-infected PrPM129 mice in cortex and cerebellum. The significance was calculated with one-way ANOVA Friedman test $(* P<0.05, * * P<0.01, * * * P<0.001)$

cortex in the SCJD MM1 subtype (Fig. 2c) and in the cerebellum of VV2 subtype as compared to age-matched control subjects (Fig. 2a, c), showing that the response was region- 
A
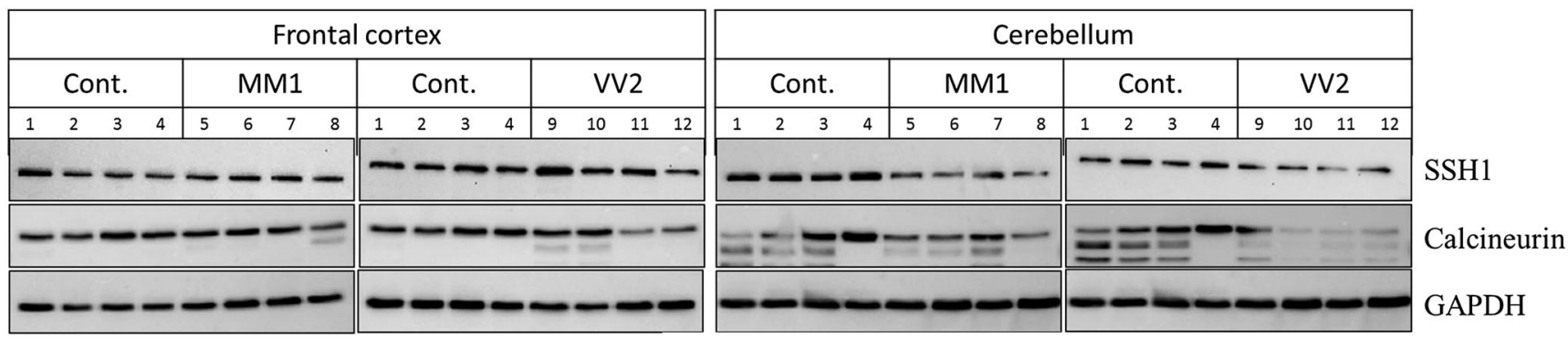

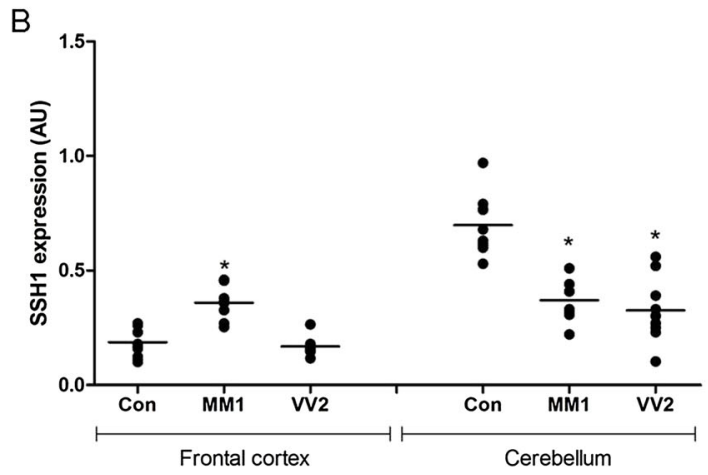

Fig. 7 SSH1 and calcineurin in SCJD MM1 and VV2 subtypes. Western blotting analysis: a SSH1 and calcineurin in four representative cases each for control, sCJD MM1, and sCJD VV2 cases; GAPDH immunostaining was used to normalize total protein loading. b, c Densitometry analysis from four independent $( \pm \mathrm{SD})$ immunoblotting

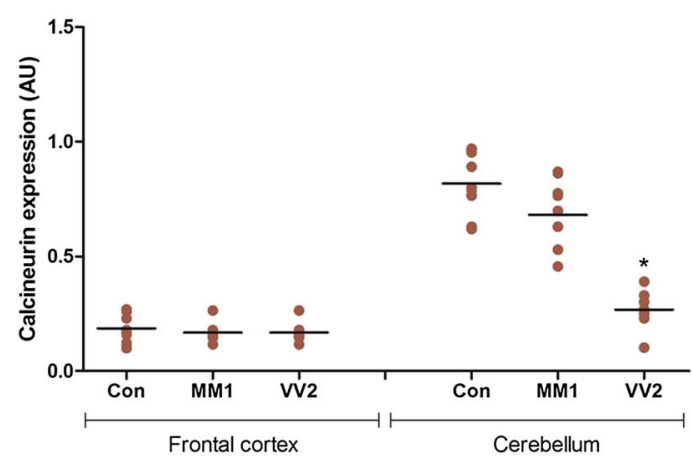

experiments of 15 control (Con), $15 \mathrm{MM} 1$, and $15 \mathrm{VV} 2$ cases. The significance was calculated with one-way ANOVA Friedman test $(* P<0.05)$. Region-dependent significantly altered expression is found in both $\mathrm{s} C \mathrm{JD}$ subtypes compared to controls. $A U$ arbitrary units

stage of the disease (from 120 to 210 dpi) (Fig. 3a, b). The p-cofilin(Ser3) levels showed upregulation in the cortex region of MM1-M129 mice as compared to VV2-Val129 mice (Fig. 3a, c-e). The cerebellum region of MM1-Met129 subtype sCJD showed a significant decrease of p-cofilin(Ser3) at pre-symptomatic stage (120 dpi) and upregulation at symptomatic stage (210 dpi) (Fig. 3d, f). In parallel, the cerebellum VV2-Val129 subtype showed increase of $\mathrm{p}$ cofilin(Ser3) only at pre-symptomatic stages (120 dpi) (Fig. 3a, f).

This early stage regulated response of phosphorylation (deactivation) of cofilin indicating that cofilin activity decreases at the pre-symptomatic stage of the disease, and to explore the mechanism of this altered activity of cofilin-1, we analyzed changes in LIMK1, the modulator of cofilin phosphorylation. The MM1-Met129 mice showed significant decrease of LIMK1 at pre-symptomatic stage of the disease (from 120 to $160 \mathrm{dpi}$ ) in cortex region of MM1-Met129 mice cortex (Fig. 4a, b); however, P-LIMK1 showed significant increase at pre-symptomatic stage of the disease (120 dpi) in cortex region of MM1-Met129 mice (Fig. 4d, e). MM1-Met129 mice cerebellum showed upregulation of LIMK1 at presymptomatic stage of the disease (120 dpi) (Fig. 4a, c), and P-LIMK1 showed significant decrease at pre-symptomatic stage of the disease (120 dpi) (Fig. 4d, f). VV2-Val129 mice cerebellum showed upregulation of LIMK1 and P-LIMK1 at pre-symptomatic stage of the disease (160 dpi) (Fig. 4a, c, f).

cerebellum region at pre-symptomatic and symptomatic 


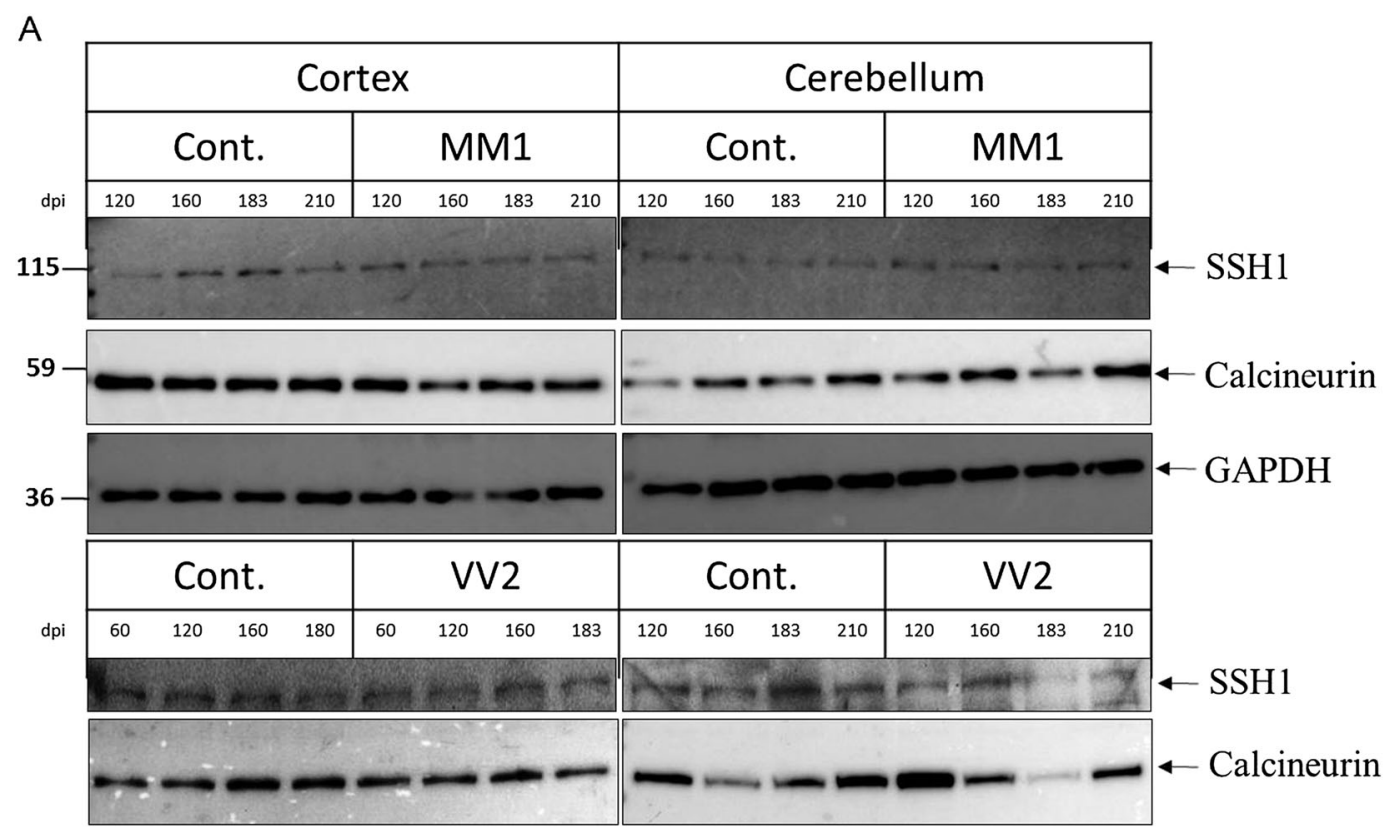

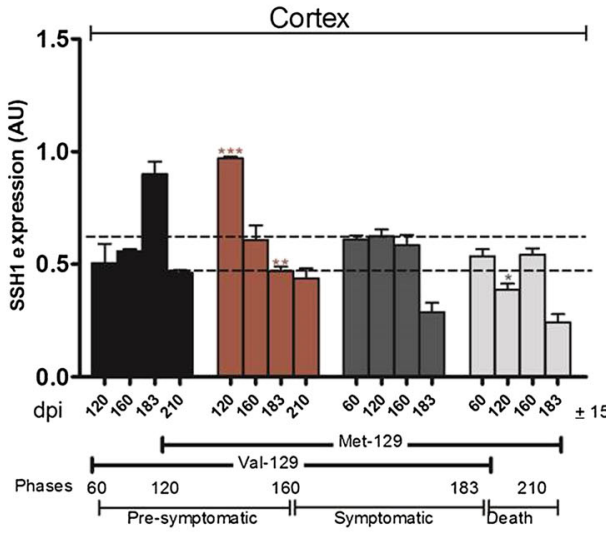

D

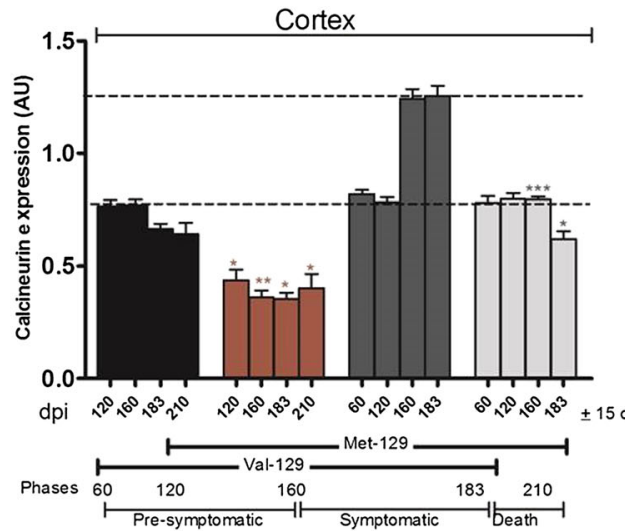

Fig. 8 Expression of SSH1 and calcineurin at pre-symptomatic and symptomatic stage of sCJD MM1 and VV2 in mice. Tg340 mice expressing about 4-fold level of human PrPM129 were infected with sCJD MM1 and also VV2 10\% brain homogenates. Samples were collected at indicated days post-infection (dpi): pre-symptomatic stage (60 dpi in VV2, 120 dpi in MM1) and symptomatic stages (120 183 dpi in VV2 and 160-210 dpi). a SSH1 and calcineurin expression

E
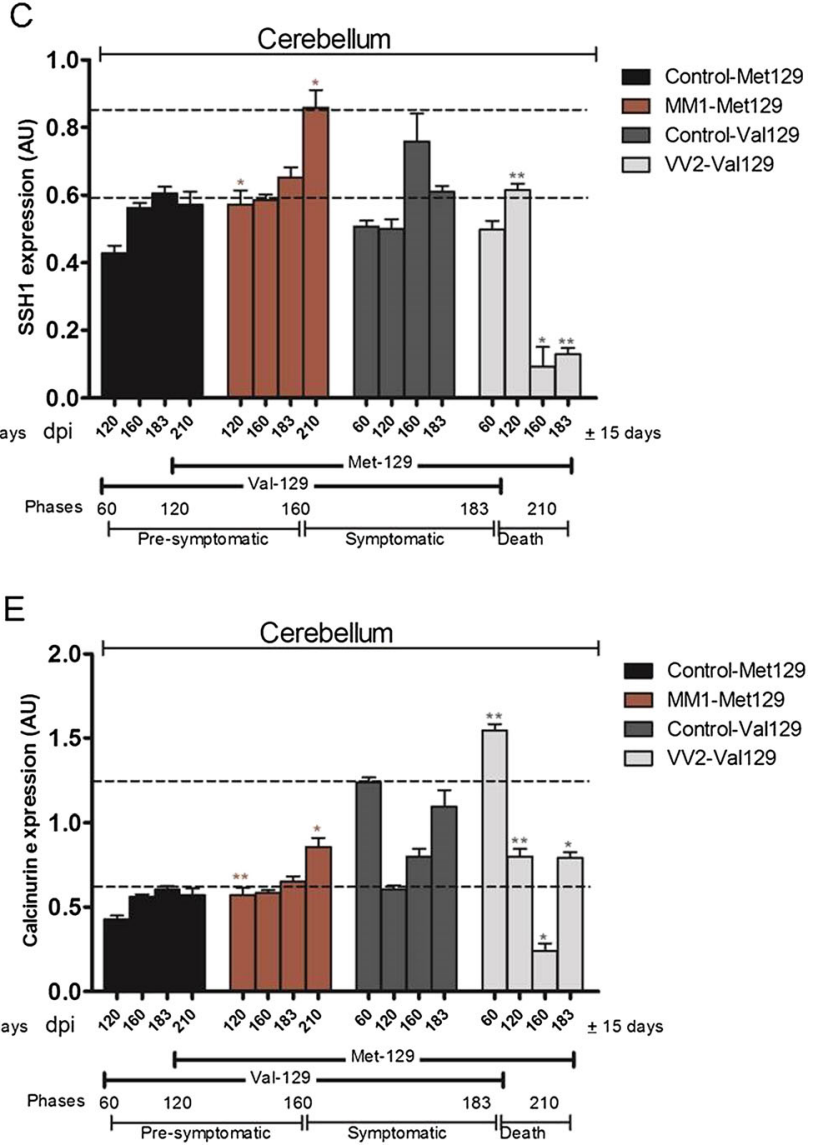

in the cortex and cerebellum were observed by Western blot analysis using SSH1 and calcineurin antibodies. b-e Densitometry analysis from four independent $( \pm \mathrm{SD})$ immunoblotting experiments of SSH1 and calcineurin from control and SCJD MM1- and VV2-infected PrPM129 mice in cortex and cerebellum. The significance was calculated with oneway ANOVA Friedman test $(* P<0.05, * * P<0.01, * * * P<0.001)$ 
Lastly, to demonstrate the kinases that regulate phosphorylation of LIMK, we found significant upregulation of Rock2 and APP at pre-symptomatic stage of the disease in MM1-Met129 and VV2-Val129 Tg340 mice (Figs. 5 and $6)$.

\section{SSH1 and Calcineurin Altered Expression in SCJD MM1 and VV2 Subtypes and in Pre-symptomatic and Symptomatic Stages of sCJD MM1 Mice}

Further, to better understand the aberrant cofilin-1 expression/ activity, we analyzed changes in cofilin phosphatase SSH1 and its upstream regulator calcineurin by Western blot in frontal cortex and cerebellum samples from SCJD cases with the subtypes MM1 and VV2, as well as age-matched controls. Total homogenates of the frontal cortex and cerebellum were analyzed using SSH1 and calcineurin monoclonal antibodies (Fig. 7a). Remarkably, in the cerebellum, SSH1 expression was significantly decreased in both subtypes MM1 and VV2 as compared to age-matched control samples (Fig. 7a, b) while SSH1 levels were increased in frontal cortex of MM1 subtype. However, calcineurin expression was significantly decreased only in VV2 subtype in the cerebellum (Fig. 7a, c) showing that the response was region-specific and codon 129 polymorphism-specific. Furthermore, sCJD MM1-inoculated mice demonstrated a significant increase in the expression of SSH1 at the pre-symptomatic stage (120 dpi), in contrary to sCJD VV2-inoculated mice showing significant decrease at pre-symptomatic stage (60 and $120 \mathrm{dpi}$ ) in the cortex (Fig. 8a, b). However, sCJD MM1-inoculated mice demonstrated a significant decrease in the expression of SSH1 at the symptomatic stage (183 dpi) in the cortex. The terminal or death stage showed no significant regulation in both subtypes of SCJD MM1- and VV2-inoculated mice (Fig. 8a, b). The cerebellum region of both subtypes of sCJD-inoculated mice showed differential regulation of SSH1 (Fig. 8a, c). The expression of calcineurin showed significant decrease in the cortex of SCJD MM1 mice at pre-symptomatic and symptomatic stages (from 120 to 210 dpi terminal stage). In contrast, in the SCJD VV2-inoculated mice, the calcineurin expression was significantly downregulated only in symptomatic stages (from 160 to 183 dpi terminal stage) (Fig. 8a, d). In cerebellum, calcineurin expression in SCJD MM1-inoculated mice showed a significant increased expression in the presymptomatic stage and symptomatic terminal stages (120 and 210 dpi terminal stage) (Fig. 8a, e). The sCJD VV2inoculated mice in the cerebellum showed a significant upregulation of calcineurin in the pre-symptomatic stages (60 and 120 dpi) (Fig. 8a, e). However, symptomatic and terminal death stages showed downregulation (160 and $183 \mathrm{dpi})$ (Fig. 8a, e).

\section{siRNA-Induced Gene Silencing of Cofilin-1 Leads to Dense Accumulation of F-Actin, Activation of Microglia, and Decreased Cell Viability}

To check the cofilin-1-dependent actin depolymerization activity, we demonstrated that cofilin-1 siRNA knockdown lead to increased expression level of F-actin and decreased expression level of $\mathrm{PrP}^{\mathrm{C}}$. Approximately $70-80 \%$ of cofilin- 1 expression depletion was achieved in PCNs (Fig. 9a, c). The immunofluorescence results demonstrated that cofilin-1 siRNA deficit cells showed increased expression level and dense accumulation of F-actin (Fig. 9a, b). However, $\operatorname{PrP}^{\mathrm{C}}$ showed decreased expression and altered localization pattern (Fig. 9a, d). The percent colocalization of cofilin-1 and F-actin showed significant decrease in the cofilin-1 depleted PCNs. However, F-actin and $\operatorname{PrP}^{\mathrm{C}}$ showed significant upregulation of percent colocalization in the cofilin-1 depleted PCNs (Fig. 9e, f).

As cofilin-1 gene deletion leads to lethality in mouse [49], further, we investigated the effect of cofilin-1 depletion on cell viability in both $\operatorname{PrP}^{\mathrm{C}}$ wild-type and knockout PCN cells. Cofilin-1 depletion resulted in decreased Cell viability in both $\operatorname{PrP}^{\mathrm{C}}$ wild-type and knockout PCN cells (Fig. 10a) while concomitantly increased caspase-3 activity (Fig. 10b). Interestingly, $\mathrm{PrP}^{\mathrm{C}}$-expressing PCNs with siRNA cofilin-1 depletion, showed more viability and less caspase- 3 activation as compared to $\mathrm{PrP}^{-/-}$PCNs depleted with cofilin-1 (Fig. 10a, b); suggesting protective role of $\operatorname{PrP}^{\mathrm{C}}$ on cell viability.

As both in vitro and in vivo studies have shown that neuronal apoptosis and neuronal death precedes microglia activation [50], so accordingly, we investigated the activation status of microglia in cofilin-1 depleted $\mathrm{PCN}$ cultures $\left(\mathrm{PrP}^{\mathrm{C}}\right.$ wildtype and $\operatorname{PrP}^{\mathrm{C}}$ knockout cells). Here, we demonstrated a correlation between presence and absence of $\operatorname{PrP}^{\mathrm{C}}$, and the cofilin-1 depletion induced microglial activation in primary cortical neurons (Fig. 10c-e). Immunofluorescence data demonstrated the different populations of microglia after siRNA against cofilin-1 treatment in PCNs (Fig. 10c) with a shift towards activated microglia in cofilin-1 depleted cells. These microglia populations showed marked differences in the expression of cofilin-1 and p-cofilin with the upregulation of $\mathrm{p}$ cofilin in activated microglia (Fig. 11a). The colocalization analysis of cofilin-1, and p-cofilin(Ser3) with Iba1 showed significant increase shift between p-cofilin and Ibal in the cofilin-1-depleted PCNs as compared to control (Fig. 11b, c). Overall, coflin-1 regional density, distribution, and/or activity of microglia and microglia-derived factors may influence the region-specific role for this cell type (Fig. 13).

Further, it is already been reported that the microglia showed upregulation in SCJD cases, with increased expression levels in the frontal cortex in MM1 and in the cerebellum in 

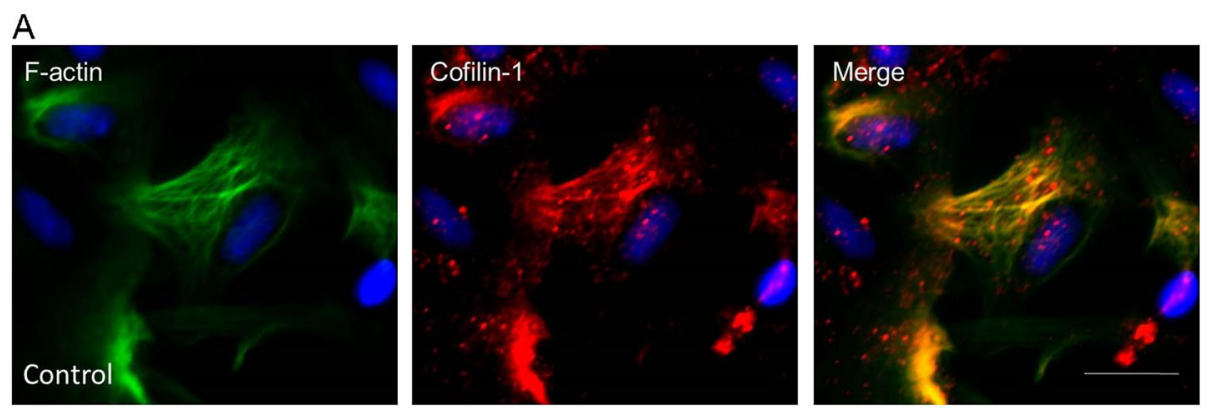

\section{B}
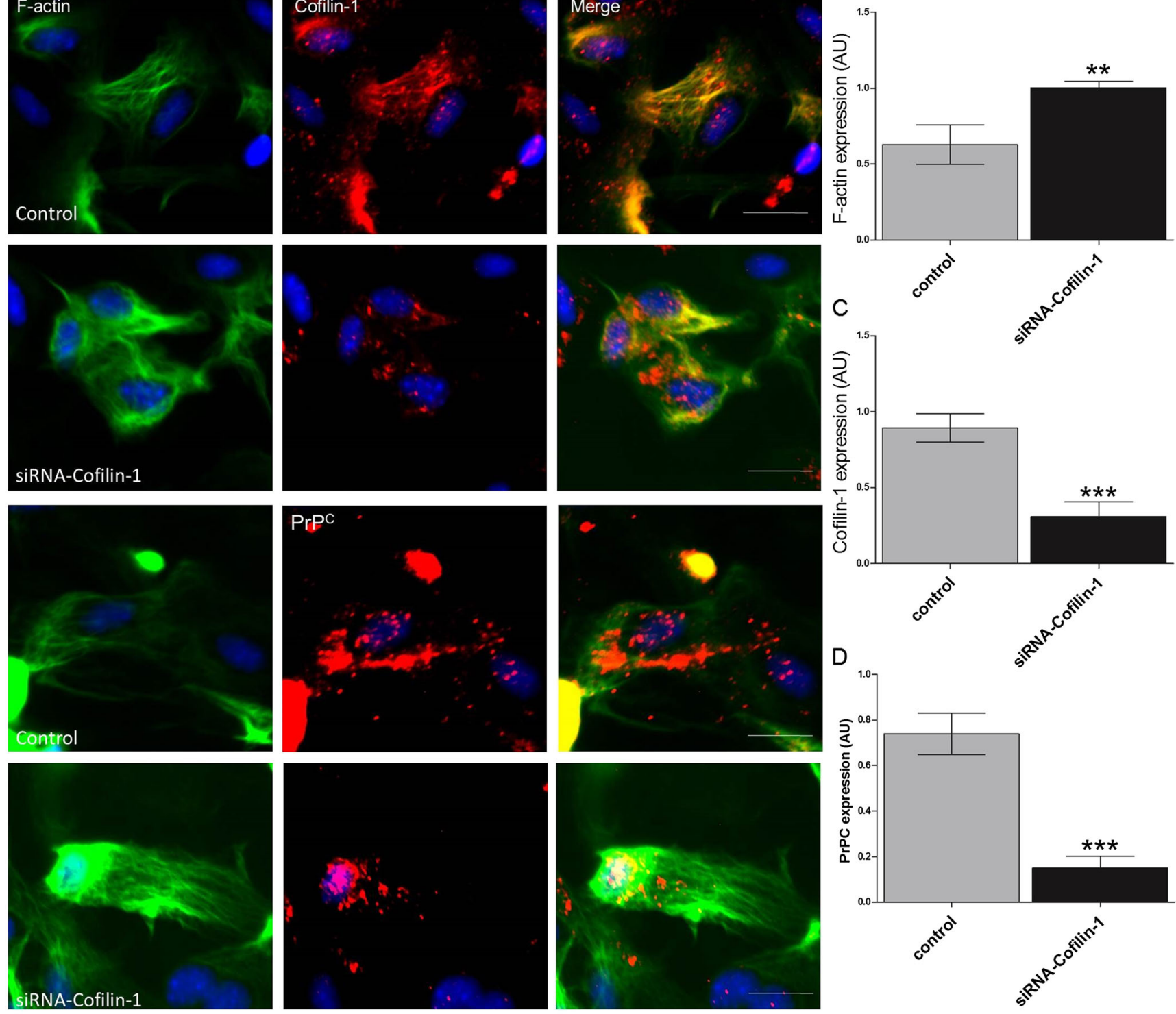

E

\begin{tabular}{|c|c|c|c|c|}
\hline Nr. & Rr & $\begin{array}{l}\text { Coloc. Coefficient } \\
\text { F-actin (M1) }\end{array}$ & $\begin{array}{l}\text { Coloc. Coefficient } \\
\text { Cofilin-1 (M2) }\end{array}$ & \% Colocalization \\
\hline Control & 0.374 & 0.789 & 0.849 & 2.18 \\
\hline siRNA-Cofilin-1 & 0.425 & 0.998 & 0.989 & 0.91 \\
\hline & & & $\begin{array}{c}\text { Coloc. Coefficient } \\
\text { PrPC(M2) }\end{array}$ & \\
\hline Control & & & & 3.01 \\
\hline siRNA-Cofilin-1 & 0.735 & & & 4.91 \\
\hline
\end{tabular}

F
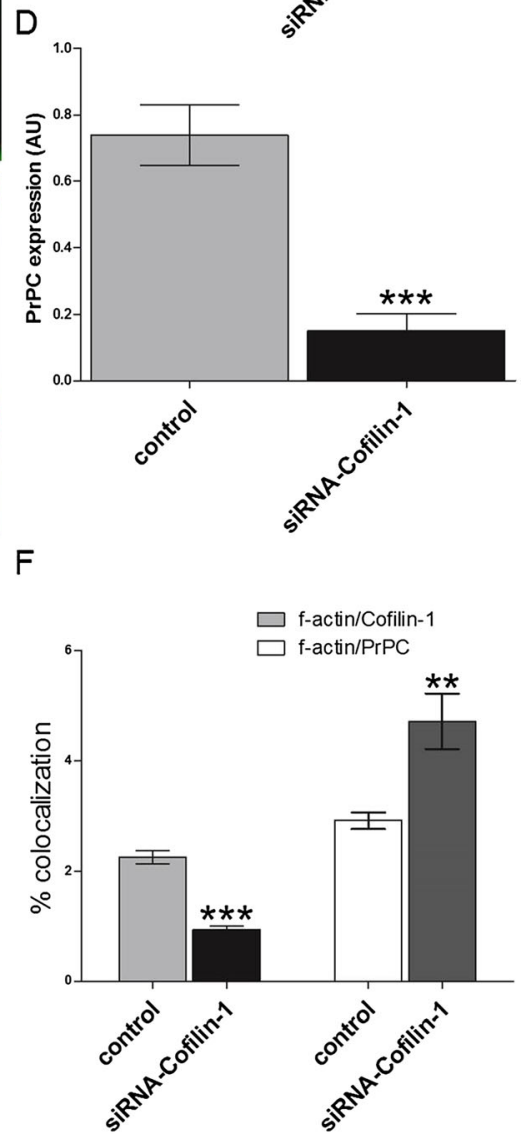

VV2 cases. In the CJD MM1 mice, the microglial marker Iba1 also showed upregulation at mRNA level [51]. Our data also showed region-specific upregulation of ibal levels, microglia marker, in SCJD cases for both subtypes MM1 and VV2 
4 Fig. 9 Colocalization of F-actin, cofilin-1, and $\operatorname{PrP}^{\mathrm{C}}$ in $\mathrm{PCNs}$ after depletion of cofilin-1. Primary cultures of mouse cortex neurons (PCNs) were prepared from pregnant mice at embryonic day 14; the mice were prnp (the PrP-encoding gene). PCNs were treated with cofilin-1 siRNA and fixed and immunostained for F-actin, cofilin-1, and $\operatorname{PrP}^{\mathrm{C}}$ examples from a single neuron showing a co-immunostaining of $\mathrm{F}$ actin and cofilin-1, F-actin, and $\operatorname{PrP}^{\mathrm{C}}$. $\mathbf{b}, \mathbf{c}, \mathbf{d}$ Quantification of images taken in different regions of neurons fixed after siRNA depletion and showed a significant $(* * P<0.01)$ increase in $\mathrm{F}$-actin expression and a decrease of cofilin- 1 expression $(* * * P<0.001)$ and $\operatorname{PrP}^{\mathrm{C}}(* * * P<0.001)$. e, f Pearson's colocalization correlation coefficient $\mathrm{rp}(-1 \leq \mathrm{rp} \leq 1)$ experiments and graph was generated by ImageJ (WCIF plugin) software. Quantification of colocalization was determined with and without cofilin1 siRNA treatment by ImageJ (WCIF plugin) software. Densitometry analysis from four independent $( \pm \mathrm{SD})$ experiments and significance was calculated with Student's $t$ test $(* * P<0.01$, *** $P<0.001)$

(Fig. 12). We also found significant upregulation of ibal expression at pre-symptomatic stage and symptomatic stage of the disease in MM1-Met129 and VV2-Val129 Tg340 mice (Fig. 12).

\section{Discussion}

\section{Selective Interactive Association Between $\operatorname{PrP}^{\mathrm{C}}$ and Cofilin-1}

We have previously shown that by using interactomic approaches, we can isolate cofilin-1 as a binding partner of $\mathrm{PrP}^{\mathrm{C}}$ in HpL3-4 cells [46]; however, the prion-specific regulatory response of cofilin-1 and its activation during prion disease progression remained to be determined. In this study, we showed that the cofilin-1 and phosphorylated form of cofilin at Ser3 interacts and co-sediment with $\operatorname{PrP}^{\mathrm{C}}$ in primary cortical and cerebellar neurons. Cofilin-1, which is a member of cofilin-actin cytoskeleton assembly and is involved in abnormal rod formation [52], specifically in cortical region of $\mathrm{AD}$ brain [52], has shown direct interaction with $\operatorname{PrP}^{\mathrm{C}}$ in our study, suggesting a role of cofilin-1 in pathogenesis of Prion diseases. Previously, cofilin1 has been shown as an interactive partner of $\operatorname{PrP}^{\mathrm{Sc}}$ [47], further supporting the notion that there might be some functional association between cofilin-1 and prion protein. Altogether, our results indicate that there is a functional correlation between $\operatorname{PrP}^{\mathrm{C}}$, $\mathrm{PrP}^{\mathrm{Sc}}$, and cofilin-1 in relation to strain-specific prion diseases and with disease progression rate.

\section{Cofilin-Phosphorylation/Dephosphorylation Pathway in SCJD MM1 and VV2 Subtypes: Early and Terminal Stage Disease Progression Association}

In different cellular processes, actin is modulated by cofilin [26], and it has been hard to demonstrate that if the cofilin rod formation has damaging effects on cells, or whether it is the modification of cofilin pathway at some other step. To uncover this condition, we have taken an approach to check the alteration in cofilin-1 upstream pathway at symptomatic stage (in humans and mice) and pre-symptomatic stage (in mice) of the disease (Fig. 13).

In our regional and temporal studies, cofilin-1 (de-phosphorylated/active form) expression at protein level was significantly altered in the frontal cortex in the sCJD MM1 subtype. Interestingly, cofilin-1 (de-phosphorylated/active form) levels were significantly decreased in the cerebellum in VV2 subtype only. Levels of phosphorylated (de-activated) form which is unable to bind to actin were significantly increased in the frontal cortex in CJD-MM1 and in the cerebellum and frontal cortex of CJD-VV2 patients, suggesting that there is an increase in the inactivated form (phosphorylated form) of cofilin-1 in diseased brain. In the case of AD, cofilin overactivation and rod formation is well known [53, 54]; in contrast, we observed cofilin inactivation (increased phosphorylation) particularly at terminal stage of the disease in CJD patients, which suggest that response is CJD-specific. The novelty of our work is to report for the first time changes in cofilin pathway in CJD, thus indicating an involvement of cofilin in lesion development in prion diseases.

Time course study in sCJD MM1 and VV2-inoculated Tg340 mice showed a significant increase of p-cofilin(Ser3) in the cortex region at pre-symptomatic and symptomatic stage of the disease (from 120 to $210 \mathrm{dpi}$ ). In parallel, the cerebellum VV2 subtype showed increase of p-cofilin(Ser3) only at pre-clinical stages (120 dpi). LIMK1; phosphorylation activator of cofilin-1 at Ser3 showed significant altered regulatory response corresponding to the cofilin phosphorylation state in cortex and cerebellum and in both MM1 and VV2 subtype-inoculated Tg340 mice.

This early stage regulated response of phosphorylation (deactivation) of cofilin-1 indicates that cofilin-1 activity decreases at the pre-symptomatic stage of the disease. LIMK1 is responsible for the phosphorylation of cofilin and is activated by phosphorylation at thr505 and th508, which showed a 2-fold increase in the phosphorylation level of kinase, which indicates a stronger LIMK activity as early disease response. Finally, among the kinases that phosphorylate LIMK is RhoA-associated kinase (Rock2) whose activity is regulated by small G protein RhoA [27-29] and amyloid precursor protein (APP). Initially, there was an increase in Rock 2 levels at $60 \mathrm{dpi}$, which return to normal at $120 \mathrm{dpi}$, and then increase again at $180 \mathrm{dpi}$, probably associated with different pattern of $\mathrm{PrP}^{\mathrm{Sc}}$ aggregates in these mice. We previously reported that the RhoA activity was regulated by phosphorylation of myosin light-chain (MLC) in association with Arfl-dependent PI3K pathway [36]. The APP showed significant upregulation at pre-symptomatic stage of the disease in MM1- and VV2-inoculated Tg340 mice. These data argue that the phosphorylation (de-activation) of cofilin at Ser3 is regulated by the upstream activation of the RhoA-Rock2-LIMK1-pathway at early stage of the disease. 

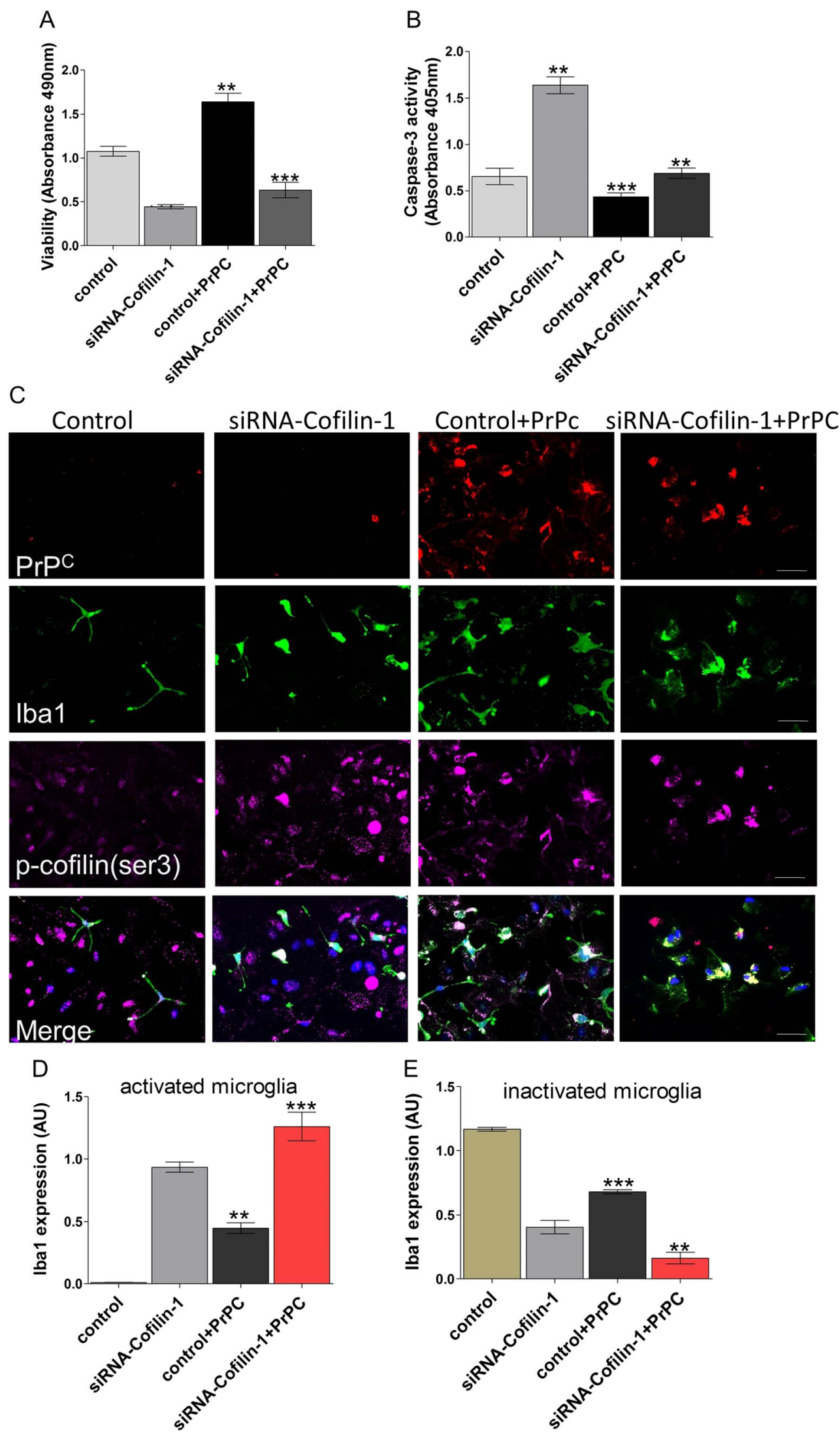

The reactivation of cofilin-1 by dephosphorylation at Ser3 is SSH1 dependent in neurons [55]. However, pathways for
p21-LIM kinase activation, protein kinase $\mathrm{C} / \mathrm{G}$, or reactive oxygen species were not responsible for these alterations 
Fig. 10 PCN viability and activation of microglia after depletion of cofilin-1. Primary cultures of mouse cortex neurons (PCNs) were prepared from pregnant mice at embryonic day 14; the mice were prnp (the PrP-encoding gene). PCNs were treated with cofilin-1 siRNA. a Cell viability was measured by MTS assay $48 \mathrm{~h}$ post transfection. The viability values are shown as absorbance at $490 \mathrm{~nm}$. Data points are the means \pm SEM of values from four different experiments. The cells expressing $\operatorname{PrP}^{\mathrm{C}}$ displayed elevated level of cell viability under cofilin-1 depletion as compared with out $\operatorname{PrP}^{\mathrm{C}}$. The significance was performed by one-way ANOVA Friedman test $(* * P<0.01$, $* * * P<0.001)$. b Caspase- 3 activity was detected by fluorescence measurement of the cleaved pNA from the substrate peptide DEVD-pNA. Data points are the means \pm SD of values from four different experiments. The cells expressing $\operatorname{PrP}^{\mathrm{C}}$ displayed decreased level of caspase- 3 cleavage activity under cofilin-1 depletion as compared with out $\mathrm{PrP}^{\mathrm{C}}$. The significance was performed by one-way ANOVA Friedman test $(* * P<0.01$, $* * * P<0.001)$. c Colocalization of $\mathrm{PrP}^{\mathrm{C}}$, Iba1, and p-cofilin(Ser3) in PCNs after depletion of cofilin-1, fixed and immunostained. d, e Quantification of images taken in different regions of neurons and showed a significant altered expression of activated and inactivated Iba1-positive microglia after siRNA depletion in the presence and absence of $\operatorname{PrP}^{C}$ expression. Quantification of colocalization was determined by ImageJ (WCIF plugin) software, and significance was calculated with Student's $t$ test $(* * P<0.01, * * * P<0.001)$
[56]. Although other kinases and phosphatases can affect cofilin-1 activity, LIMK1 and SSH1 show the highest substrate specificity [26]. Remarkably, phosphorylation at serines 937 and 978 inactivates SSH1 [30]. Further, not only cofilin-1 but also LIMK1 is dephosphorylated by SSH1 activity. So, SSH1 can control cofilin-1 activity either directly or indirectly through an upstream regulation of LIMK1 [30].

To further explore the possible mechanism responsible for altered expression/activity of cofilin-1, we measured changes in cofilin phosphatase SSH1 and its upstream regulator calcineurin and found the altered response at early disease presymptomatic stage in both subtypes. Calcineurin is responsible for cofilin dephosphorylation either directly or indirectly via an intermediary phosphatase SSH1. Calcineurin has been implicated in the regulation of synaptic plasticity and memory [57, 58]. Hyperactivation of calcineurin leads to synaptic abnormalities and neuronal death [59]. On the other side, SSH1 also deactivates LIMK1 to regulate cofilin activation state and could be the risk factor for the modulation of the whole pathway. The mechanism responsible for cofilin-1 activation or inactivation/

\section{A}
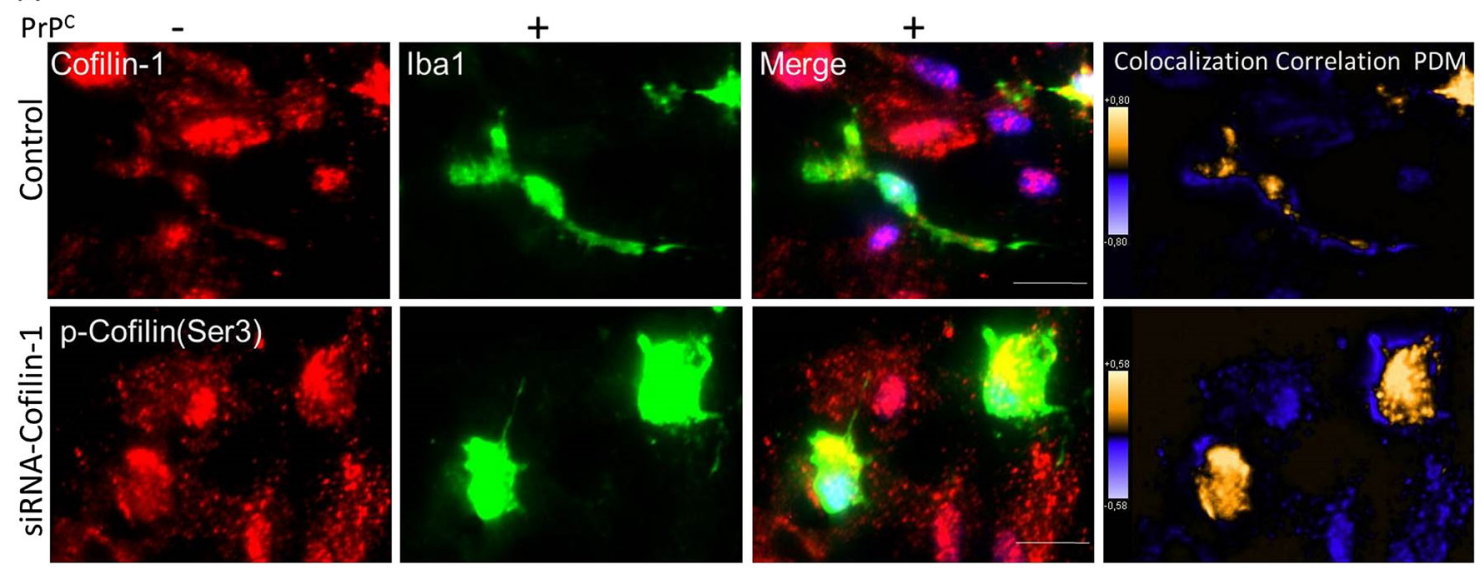

B

C

\begin{tabular}{|c|l|c|c|c|}
\hline Nr. & Rp & $\begin{array}{l}\text { Coloc. Coefficient p- } \\
\text { cofilin-1 (M1) }\end{array}$ & $\begin{array}{l}\text { Coloc. } \\
\text { Coefficient Iba1 } \\
\text { (M2) }\end{array}$ & $\begin{array}{l}\text { Colocalization } \\
\text { Col }\end{array}$ \\
\hline 1. & 0.247 & 0.789 & 0.849 & 0.48 \\
\hline 2. & 0.425 & 0.998 & 0.989 & 3.78 \\
\hline
\end{tabular}

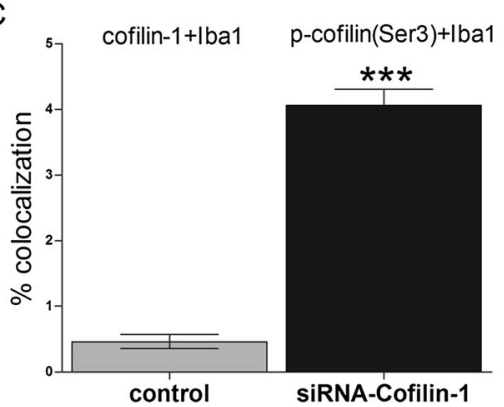

Fig. 11 Colocalization of cofilin-1 and p-cofilin(Ser3) in PCNs after depletion of cofilin-1. Primary cultures of mouse cortex neurons (PCNs) were prepared from pregnant mice at embryonic day 14; the mice were prnp (the PrP-encoding gene). PCNs were treated with cofilin-1 siRNA and fixed and a co-immunostained for cofilin-1 and pcofilin(Ser3). b, c Quantification of images taken in different regions of neurons fixed after siRNA depletion and showed a significant redistribution of $\mathrm{p}$-cofilin(Ser3) in activated form of Iba1-positive microglia. Pearson's colocalization correlation coefficient $\mathrm{rp}$ $(-1 \leq \mathrm{rp} \leq 1)$ experiments and graph were generated by ImageJ (WCIF plugin) software. Quantification of colocalization that was determined with and without cofilin-1 siRNA treatment showed significant increase of p-cofilin(Ser3) in Iba1-positive microglia. Densitometry analysis from four independent $( \pm \mathrm{SD})$ and significance was calculated with Student's $t$ test $(* * * P<0.001)$ 

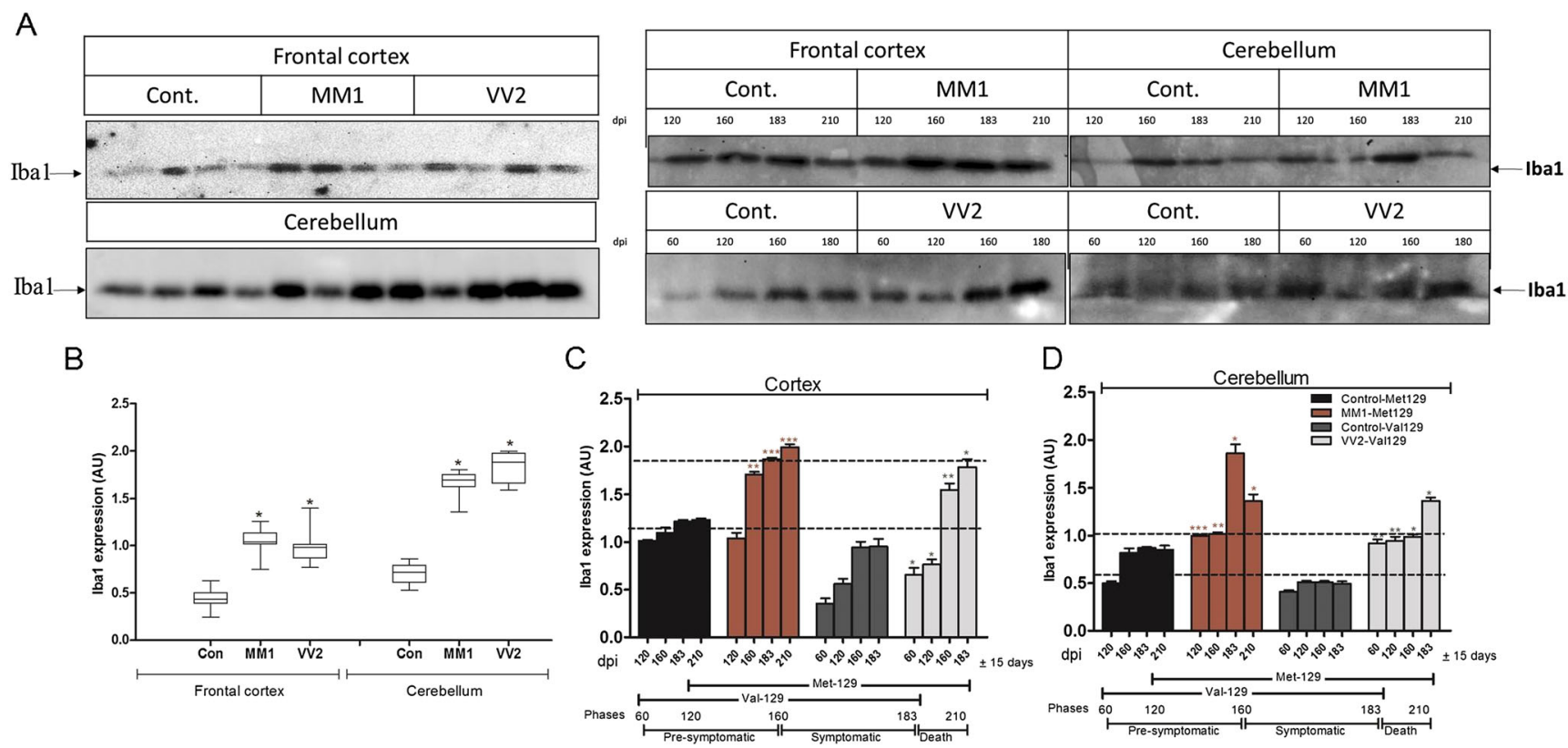

Fig. 12 Iba1 expression in SCJD MM1 and VV2 subjects and at presymptomatic and symptomatic stage of sCJD MM1 and VV2 mice. Western blotting analysis: a Iba1 protein expression in four representative cases each for control, sCJD MM1, and SCJD VV2 cases and in Tg340 mice infected with sCJD MM1 and also VV2 10\% brain homogenates. Samples were collected at indicated days post-infection (dpi): pre-symptomatic stage (60 dpi in VV2 120 dpi in MM1) and symptomatic stages (120-183 dpi in VV2 and 160-210 dpi). b

phosphorylation may be different in both subtypes and brain regions and dependent, at least in part, on $\operatorname{PrP}^{\mathrm{Sc}}$ aggregates.

\section{Dense Accumulation of F-Actin and Activation of Microglia}

The activation of cofilin-1 (dephosphorylation) is a key factor for the dynamics in actin depolymerization and cytoskeleton assembly [60]. Cofilin phosphorylation was aberrantly upregulated in the terminal stage of the disease, in parallel to the
Densitometry analysis from four independent $( \pm \mathrm{SD})$ immunoblotting experiments of 15 control (Con), $15 \mathrm{MM} 1$, and $15 \mathrm{VV} 2$ cases. c, d Densitometry analysis from four independent $( \pm \mathrm{SD})$ immunoblotting experiments Iba1 from control and sCJD MM1- and VV2-infected PrPM129 mice in cortex and cerebellum. The significance was calculated with one-way ANOVA Friedman test $(* P<0.05)$. Regiondependent significantly altered expression is found in both SCJD subtypes compared to controls. $A U$ arbitrary units

reduction of the SSH1. Interestingly, our findings indicate that cofilin-1 depletion leads to denser actin filaments corresponding to repression of level of $\mathrm{PrP}^{\mathrm{C}}$ in $\mathrm{PCN}$. The reason for decreased levels of $\mathrm{PrP}^{\mathrm{C}}$ might be altered actin dynamics leading to proteasome degradation of $\operatorname{PrP}^{\mathrm{C}}$ instead of its transportation to the cell membrane due to lack of cofilin-1-dependent remodeling of actin cytoskeleton [61] as the localization pattern of $\operatorname{PrP}^{\mathrm{C}}$ was also altered in cofilin-1 depleted cells. Several recent studies also support the idea that alterations in cofilin activation state contribute to actin pathology in neurodegeneration.
Fig. 13 Schematic representation of molecular events of cofilinLIMK-SSH1-dependent actin assembly showed altered response at pre-symptomatic stage and symptomatic stage of the disease. Yellow highlighted area presents alterations demonstrated in this study (color figure online)

\section{Prion pre-clinical stage}

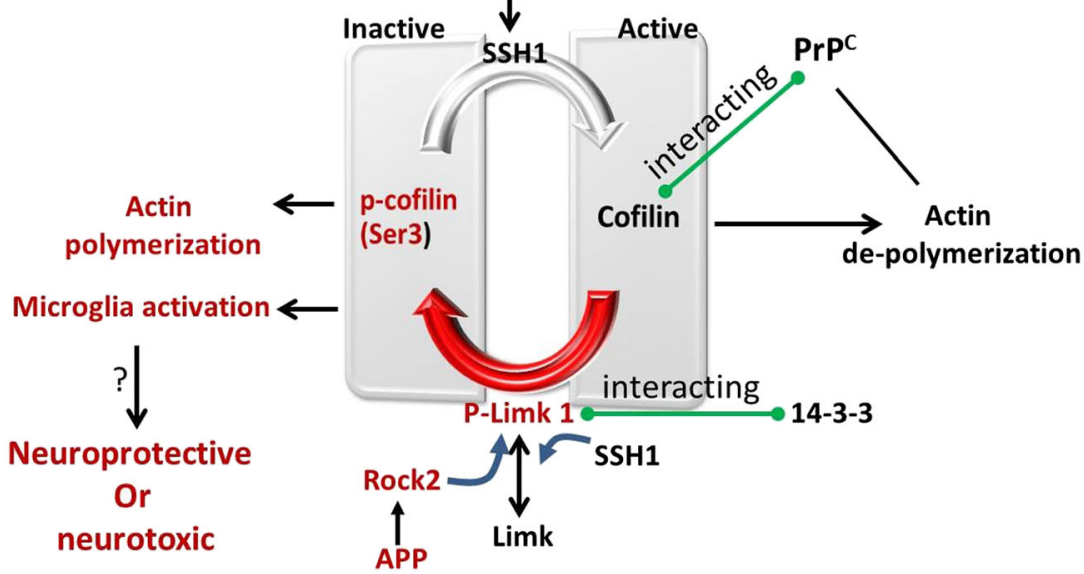


The activation of microglial is a trademark of the neuroimmunological response in prion disease [62]. Recent report demonstrated the suggestive role of microglia during the clearance of cofilin aggregation [53]. Our data demonstrated different populations of microglia in response to depletion of cofilin- 1 and presence of $\operatorname{PrP}^{\mathrm{C}}$. The different activation statuses of microglia after depletion of cofilin-1 demonstrate a link between cofilin-1, $\operatorname{PrP}^{\mathrm{C}}$, and microglia activation during the course of prion disease. These different microglia populations showed marked differences in the expression of dephosphorylated form (active form) in non-active microglia and phosphorylated form (inactivated) of cofilin in activated form of microglia.

However, the upregulated microglia expression showed disease subtype and brain region-specific response in CJD patients (Fig. 12). CJD-MM1 patients showed significantly and specifically upregulated microglia in frontal cortex; however, CJD-VV2 subtype patients showed this response specifically in cerebellum [51].

Altogether, our data demonstrated the different populations of microglia in response to depletion of cofilin-1, presence of $\operatorname{PrP}^{\mathrm{C}}$, and disease subtype manner. These microglia populations showed marked differences in the expression of dephosphorylated form of cofilin in non-active microglia and phosphorylated form of cofilin in activated microglia. Cofilin-1 regional density, distribution, and/or activity of microglia and microglia-derived factors may influence the regionspecific role for this cell type.

This regional and subtype-specific post-translational altered pattern of cofilin-LIMK-APP pathway in SCJD MM1 and VV2 and a modified temporal response in SCJD MM1 and VV2 mice could correlate with the disease severity and progression in neurons in correlation with microglia. The demonstration in respect with subtype manner could prove to be the cardinal specific targets for therapeutic intervention.

\section{Conclusion}

In conclusion, this study demonstrates altered cofilin-LIMKSSH1 pathway in sporadic CJD-MM1 and VV2 subtypes at early disease stage in a strain-specific subtype manner. The regulated response may contribute actin cytoskeleton abnormalities that ultimately lead to synapse and dendritic spine loss, as actin dynamics are responsible for synapse formation, maturation, and stability and for maintenance of dendritic spines in CJD. Taken together, our findings highlight a key pre-and early symptomatic role of cofilin- 1 activation in neurodegeneration specifically as an early regulatory response, suggesting the idea that targeting the cofilin signaling axis might be a promising strategy and a potential early diagnostic approach useful to reveal therapeutic strategy for misfolded protein differential response.
Acknowledgements We give special thanks to Dr. Torres at CISA INIA who produced the tg340 mice.

Compliance with Ethical Standards Human samples from the Institute of Neuropathology Brain Bank (HUB-ICO-IDIBELL Biobank) and Biobank of Hospital Clinic-IDIBAPS were obtained following the Spanish legislation (Ley de la Investigación Biomédica 2013 and Real DecretoBiobancos 2014) and the approval of the local ethics committees.

All animal experiments were performed in accordance with the ethical standard set by Regierungspräsidium Tübingen (Regional Council) Experimental No. FLI 231/07 file reference number 35/9185.81-2. All animal experiments have been performed in compliance with the institutional and French national guidelines, in accordance with the European Community Council Directive 86/609/EEC. The experimental protocol was approved by the INRA Toulouse/ENVT ethics committee.

\section{References}

1. Tschampa HJ, Kallenberg K, Kretzschmar HA, Meissner B, Knauth M, Urbach H, Zerr I (2007) Pattern of cortical changes in sporadic Creutzfeldt-Jakob disease. Am J Neuroradiol 28:1114-1118

2. Bishop MT, Will RG, Manson JC (2010) Defining sporadic Creutzfeldt-Jakob disease strains and their transmission properties. Proc Natl Acad Sci 107:12005-12010

3. Parchi P, Giese A, Capellari S, Brown P, Schulz-Schaeffer W, Windl O, Zerr I, Budka H et al (1999) Classification of sporadic Creutzfeldt-Jakob disease based on molecular and phenotypic analysis of 300 subjects. Ann Neurol 46:224-233

4. Parchi P, Strammiello R, Notari S, Giese A, Langeveld JP, Ladogana A, Zerr I, Roncaroli F et al (2009) Incidence and spectrum of sporadic Creutzfeldt-Jakob disease variants with mixed phenotype and co-occurrence of PrPSc types: an updated classification. Acta Neuropathol 118:659-671

5. Ferrer I, Puig B, Blanco R, Marti E (2000) Prion protein deposition and abnormal synaptic protein expression in the cerebellum in Creutzfeldt-Jakob disease. Neuroscience 97:715-726

6. Jeffrey M, Halliday WG, Bell J, Johnston AR, Mac Leod NK, Ingham C, Sayers AR, Brown DA et al (2000) Synapse loss associated with abnormal PrP precedes neuronal degeneration in the scrapie-infected murine hippocampus. Neuropathol Appl Neurobiol 26:41-54

7. Jeffrey M, McGovern G, Sisó S, González L (2011) Cellular and sub-cellular pathology of animal prion diseases: relationship between morphological changes, accumulation of abnormal prion protein and clinical disease. Acta Neuropathol 121:113-134

8. Loubet D, Dakowski C, Pietri M, Pradines E, Bernard S, Callebert J, Ardila-Osorio H, Mouillet-Richard S et al (2012) Neuritogenesis: the prion protein controls beta1 integrin signaling activity. FASEB J 26:678-690

9. Zafar S, Younas N, Correia S, Shafiq M, Tahir W, Schmitz M, Ferrer I, Andreoletti O et al (2016) Strain-specific altered regulatory response of Rab7a and Tau in Creutzfeldt-Jakob disease and Alzheimer's disease. Mol Neurobiol

10. Eiseler T, Hausser A, De Kimpe L, Van Lint J, Pfizenmaier K (2010) Protein kinase D controls actin polymerization and cell motility through phosphorylation of cortactin. J Biol Chem 285: 18672-18683

11. Munnamalai V, Weaver CJ, Weisheit CE, Venkatraman P, Agim ZS, Quinn MT, Suter DM (2014) Bidirectional interactions between NOX2-type NADPH oxidase and the F-actin cytoskeleton in neuronal growth cones. J Neurochemn/a

12. Yamada H, Abe T, Satoh A, Okazaki N, Tago S, Kobayashi K, Yoshida Y, Oda Y et al (2013) Stabilization of actin bundles by a 
dynamin $1 /$ cortactin ring complex is necessary for growth cone filopodia. J Neurosci 33:4514-4526

13. Bosch M, Castro J, Saneyoshi T, Matsuno H, Sur M, Hayashi Y (2014) Structural and molecular remodeling of dendritic spine substructures during long-term potentiation. Neuron Cell Press 82: 444-459

14. Okamoto K, Nagai T, Miyawaki A, Hayashi Y (2004) Rapid and persistent modulation of actin dynamics regulates postsynaptic reorganization underlying bidirectional plasticity. Nat Neurosci 7: 1104-1112

15. Schubert V, Dotti CG (2007) Transmitting on actin: synaptic control of dendritic architecture. J Cell Sci 120:205-212

16. Sekino Y, Kojima N, Shirao T (2007) Role of actin cytoskeleton in dendritic spine morphogenesis. Neurochem Int 51:92-104

17. Star EN, Kwiatkowski DJ, Murthy VN (2002) Rapid turnover of actin in dendritic spines and its regulation by activity. Nat Neurosci 5:239-246

18. Bravo-Cordero JJ, Magalhaes MA, Eddy RJ, Hodgson L, Condeelis J (2013) Functions of cofilin in cell locomotion and invasion. Nat Rev Mol Cell Biol 14:405-415

19. Hagedorn EJ, Kelley LC, Naegeli KM, Wang Z, Chi Q, Sherwood DR (2014) ADF/cofilin promotes invadopodial membrane recycling during cell invasion in vivo. J Cell Biol 204:1209-1218

20. Vitriol EA, Wise AL, Berginski ME, Bamburg JR, Zheng JQ (2013) Instantaneous inactivation of cofilin reveals its function of F-actin disassembly in lamellipodia. Mol Biol Cell 24:2238-2247

21. Kim T, Vidal GS, Djurisic M, William CM, Birnbaum ME, Garcia KC, Hyman BT, Shatz CJ (2013) Human LilrB2 is a beta-amyloid receptor and its murine homolog PirB regulates synaptic plasticity in an Alzheimer's model. Science 341:1399-1404

22. Bellenchi GC, Gurniak CB, Perlas E, Middei S, Ammassari-Teule M, Witke W (n.d.)

23. Goodson M, Rust MB, Witke W, Bannerman D, Mott R, Ponting CP, Flint J (2012) Cofilin-1: a modulator of anxiety in mice. PLoS Genet 8:e1002970

24. Gu J, Lee CW, Fan Y, Komlos D, Tang X, Sun C, Yu K, Hartzell $\mathrm{HC}$ et al (2010) ADF/cofilin-mediated actin dynamics regulate AMPA receptor trafficking during synaptic plasticity. Nat Neurosci 13:1208-1215

25. Rust MB, Gurniak CB, Renner M, Vara H, Morando L, Gorlich A, Sassoe-Pognetto M, Banchaabouchi MA et al (2010) Learning, AMPA receptor mobility and synaptic plasticity depend on ncofilin-mediated actin dynamics. EMBO J 29:1889-1902

26. Van Troys M, Huyck L, Leyman S, Dhaese S, Vandekerkhove J1, Ampe C (2008) Ins and outs of ADF/cofilin activity and regulation. Eur J Cell Biol

27. Gu H, Yu SP, Gutekunst CA, Gross RE, Wei L (2013) Inhibition of the Rho signaling pathway improves neurite outgrowth and neuronal differentiation of mouse neural stem cells. Int J Physiol Pathophysiol Pharmacol 5:11-20

28. Huang TY, Minamide LS, Bamburg JR, Bokoch GM (2008) Chronophin mediates an ATP-sensing mechanism for cofilin dephosphorylation and neuronal cofilin-actin rod formation. Dev Cell 15:691-703

29. Nishita M, Tomizawa C, Yamamoto M, Horita Y, Ohashi K, Mizuno K (2005) Spatial and temporal regulation of cofilin activity by LIM kinase and Slingshot is critical for directional cell migration. J Cell Biol 171:349-359

30. Mizuno K (2013) Signaling mechanisms and functional roles of cofilin phosphorylation and dephosphorylation. Cell Signal 25: 457-469

31. Kligys K, Yao J, Yu D, Jones JCR (2009) 14-3-3 zeta/tau heterodimers regulate Slingshot activity in migrating keratinocytes. Biochem Biophys Res Commun 383:450-454

32. Ladogana A, Sanchez-Juan P, Mitrova E, Green A, CuadradoCorrales N, Sanchez-Valle R, Koscova S, Aguzzi A et al (2009)
Cerebrospinal fluid biomarkers in human genetic transmissible spongiform encephalopathiesa. J Neurol 256:1620-1628

33. Schmitz M, Ebert E, Stoeck K, Karch A, Collins S, Calero M, Sklaviadis T, Laplanche JL et al (2015) Validation of 14-3-3 protein as a marker in sporadic Creutzfeldt-Jakob disease diagnostic. Mol Neurobiol

34. Zerr I, Bodemer M, Weber T (1997) The 14-3-3 brain protein and transmissible spongiform encephalopathy. N Engl J Med 336:874 875

35. Llorens F, Ansoleaga B, Garcia-Esparcia P, Zafar S, Grau-Rivera O, Lopez-Gonzalez I, Blanco R, Carmona M et al (2013) PrP mRNA and protein expression in brain and $\operatorname{PrP}(\mathrm{c})$ in CSF in CreutzfeldtJakob disease MM1 and VV2. Prion 7:383-393

36. Zafar S, Schmitz M, Younus N, Tahir W, Shafiq M, Llorens F, Ferrer I, Andeoletti O et al (2015) Creutzfeldt-Jakob disease subtype-specific regional and temporal regulation of ADP ribosylation factor-1-dependent rho/MLC pathway at pre-clinical stage. J Mol Neurosci 56:329-348

37. Padilla D, Beringue V, Espinosa JC, Andreoletti O, Jaumain E, Reine F, Herzog L, Gutierrez-Adan A et al (2011) Sheep and goat BSE propagate more efficiently than cattle BSE in human PrP transgenic mice. PLoS Pathog 7:e1001319

38. Carimalo J, Cronier S, Petit G, Peyrin JM, Boukhtouche F, Arbez N, Lemaigre-Dubreuil Y, Brugg B et al (2005) Activation of the JNK-c-Jun pathway during the early phase of neuronal apoptosis induced by PrP106-126 and prion infection. Eur J Neurosci 21: 2311-2319

39. Bueler H, Fischer M, Lang Y, Bluethmann H, Lipp HP, DeArmond SJ, Prusiner SB, Aguet M et al (1992) Normal development and behaviour of mice lacking the neuronal cell-surface PrP protein. Nature 356:577-582

40. Kawamoto JC, Barrett JN (1986) Cryopreservation of primary neurons for tissue culture. Brain Res 384:84-93

41. Knusel B, Michel PP, Schwaber JS, Hefti F (1990) Selective and nonselective stimulation of central cholinergic and dopaminergic development in vitro by nerve growth factor, basic fibroblast growth factor, epidermal growth factor, insulin and the insulinlike growth factors I and II. J Neurosci 10:558-570

42. Hotulainen P, Paunola E, Vartiainen MK, Lappalainen P (2005) Actin-depolymerizing factor and cofilin-1 play overlapping roles in promoting rapid $\mathrm{F}$-actin depolymerization in mammalian nonmuscle cells. Mol Biol Cell 16:649-664

43. Arrasate M, Mitra S, Schweitzer ES, Segal MR, Finkbeiner S (2004) Inclusion body formation reduces levels of mutant huntingtin and the risk of neuronal death. Nature 431:805-810

44. Zafar S, Asif AR, Ramljak S, Tahir W, Schmitz M, Zerr I (2014) Anchorless 23-230 PrP(C) interactomics for elucidation of $\operatorname{PrP}(\mathrm{C})$ protective role. Mol Neurobiol 49:1385-1399

45. Cory AH, Owen TC, Barltrop JA, Cory JG (1991) Use of an aqueous soluble tetrazolium/formazan assay for cell growth assays in culture. Cancer Commun 3:207-212

46. Zafar S, von Ahsen N, Oellerich M, Zerr I, Schulz-Schaeffer WJ, Armstrong VW, Asif AR (2011) Proteomics approach to identify the interacting partners of cellular prion protein and characterization of Rab7a interaction in neuronal cells. J Proteome Res 10:3123 3135

47. Giorgi A, Di FL, Principe S, Mignogna G, Sennels L, Mancone C, Alonzi T, Sbriccoli M et al (2009) Proteomic profiling of PrP27-30enriched preparations extracted from the brain of hamsters with experimental scrapie. Proteomics 9:3802-3814

48. Nebl G, Meuer SC, Samstag Y (1996) Dephosphorylation of serine 3 regulates nuclear translocation of cofilin. J Biol Chem 271(42): 26276-26280

49. Gurniak CB, Perlas E, Witke W (2005) The actin depolymerizing factor $\mathrm{n}$-cofilin is essential for neural tube morphogenesis and neural crest cell migration. Dev Biol 278:231-241 
50. Giese A, Brown DR, Groschup MH, Feldmann C, Haist I, Kretzschmar HA (1998) Role of microglia in neuronal cell death in prion disease. Brain Pathol 8:449-457

51. Llorens F, Lopez-Gonzalez I, Thune K, Carmona M, Zafar S, Andreoletti O, Zerr I, Ferrer I (2014) Subtype and regionalspecific neuroinflammation in sporadic Creutzfeldt-Jakob disease. Front Aging Neurosci 6:198

52. Minamide LS, Striegl AM, Boyle JA, Meberg PJ, Bamburg JR (2000) Neurodegenerative stimuli induce persistent ADF/cofilinactin rods that disrupt distal neurite function. Nat Cell Biol 2: 628-636

53. Rahman T, Davies DS, Tannenberg RK, Fok S, Shepherd C, Dodd PR, Cullen KM, Goldsbury C (2014a) Cofilin rods and aggregates concur with tau pathology and the development of Alzheimer's disease. J Alzheimers Dis 42:1443-1460

54. Woo JA, Zhao X, Khan H, Penn C, Wang X, Joly-Amado A, Weeber E, Morgan D et al (2015) Slingshot-cofilin activation mediates mitochondrial and synaptic dysfunction via Abeta ligation to beta1-integrin conformers. Cell Death Differ 22:921-934

55. Bernstein BW, Bamburg JR (2010) ADF/cofilin: a functional node in cell biology. Trends Cell Biol 20:187-195

56. Liu L, Li J, Zhang L, Zhang F, Zhang R, Chen X, Brakebusch C, Wang Z et al (2015) Cofilin phosphorylation is elevated after F- actin disassembly induced by Rac1 depletion. Biofactors 41:352359

57. Cottrell JR, Levenson JM, Kim SH, Gibson HE, Richardson KA, Sivula M, Li B, Ashford CJ et al (2013) Working memory impairment in calcineurin knock-out mice is associated with alterations in synaptic vesicle cycling and disruption of high-frequency synaptic and network activity in prefrontal cortex. J Neurosci 33:1093810949

58. Zeng H, Chattarji S, Barbarosie M, Rondi-Reig L, Philpot BD, Miyakawa T, Bear MF, Tonegawa S (2001) Forebrain-specific calcineurin knockout selectively impairs bidirectional synaptic plasticity and working/episodic-like memory. Cell 107:617-629

59. Mukherjee A, Soto C (2011) Role of calcineurin in neurodegeneration produced by misfolded proteins and endoplasmic reticulum stress. Curr Opin Cell Biol 23:223-230

60. Gohla A, Bokoch GM (2002) 14-3-3 regulates actin dynamics by stabilizing phosphorylated cofilin. Curr Biol 12:1704-1710

61. Palmer KJ, Watson P, Stephens DJ (2005) The role of microtubules in transport between the endoplasmic reticulum and Golgi apparatus in mammalian cells. Biochem Soc Symp 1-13.

62. Striebel JF, Race B, Carroll JA, Phillips K, Chesebro B (2016) Knockout of fractalkine receptor, $\mathrm{Cx} 3 \mathrm{cr} 1$, does not alter disease or microglial activation in prion-infected mice. J Gen Virol 